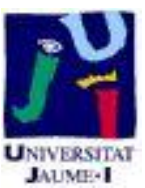

Título artículo / Títol article: The role of GC-MS/MS with triple quadrupole in pesticide residue analysis in food and the environment

Autores / Autors

F. Hernández*, M. I. Cervera, T. Portolés, J. Beltrán and E. Pitarch

Revista:

Analytical methods, n. 5 (2013)

Versión / Versió:

Preprint de l'autor

Cita bibliográfica / Cita

HERNÁNDEZ, F.,; CERVERA, M. I..; PORTOLÉS, bibliogràfica (ISO 690):

T.; BELTRÁN, J.; PITARCH, E.. Emotional eating and food intake after sadness and joy. Analytical methods, n. 5 (2103), p. 5875-5894

url Repositori UJI:

http://repositori.uji.es/xmlui/handle/10234/94270 


\title{
The role of GC-MS/MS with triple quadrupole in pesticide residue analysis in food and the environment
}

\author{
F. Hernández*, M. I. Cervera, T. Portolés, J. Beltrán and E. Pitarch \\ Research Institute for Pesticides and Water, University Jaume I, 12071 Castellón, Spain
}

\begin{abstract}
Gas chromatography coupled to tandem mass spectrometry (GC-MS/MS) using a triple quadrupole (QqQ) analyzer has in the last few years become a powerful technique for the determination of pesticide residues due to its robustness, and excellent sensitivity and selectivity. This review gives an overview of currently published applications of GCMS/MS with a QqQ analyzer for pesticide residue analysis of different food and environmental sample matrices. This technique allows the reliable quantification and identification of low pesticide concentrations for non-polar (semi) volatile compounds belonging to different chemical families. It has allowed a notable improvement of methods performance in comparison with the traditional GC methods with single stage quadrupole MS.
\end{abstract}

\section{Introduction}

Pesticide residue analysis (PRA) of food and environmental materials has become an important specialized field of modern analytical chemistry. The necessity of advanced analytical methods for its application in monitoring programs that ensure food-safety and environmentally responsible agricultural practices has been frequently highlighted. Reliable and sensitive analytical methods able to reach the low limits of quantification (LOQ) required by the legislation are needed. In most cases, LOQs lower than $0.01 \mathrm{mg}$ $\mathrm{kg}^{-1}$ in food and lower than $0.1 \mu \mathrm{g} \mathrm{L}^{-1}$ in water are needed for monitoring purposes, where the reliable identification and quantification of hundreds of pesticide residues in many different matrices is normally pursued. In recent years, new developments in sample preparation and instrumentation, especially dealing with chromatographic 
techniques coupled to mass spectrometry (MS) or tandem MS, have allowed the high quality standards required from a qualitative and quantitative point of view in PRA to be achieved.

In the past decades, gas chromatography coupled to mass spectrometry (GC-MS) methods have been mostly based on selected ion monitoring (SIM) or full scan modes, evolving from single quadrupole (Q) to ion trap (IT) analysers. The first papers dealing with PRA by GC-MS can be traced back to 1970's when the determination of a reduced number of pesticides was carried out using packed column GC systems coupled to mass spectrometers with single quadrupole analyzers. In a recent review, ${ }^{1}$ it has been reported that the single quadrupole is still the most used analyzer in combination with GC.

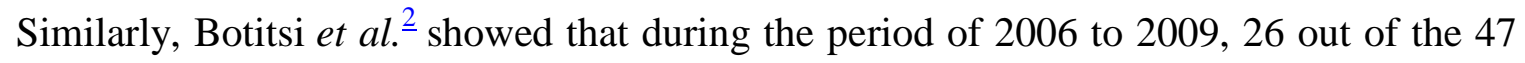
reviewed papers that employed GC-MS for the determination of pesticides in food and water were based on single quadrupole analysis. According to data reported, only 9 papers dealt with the use of triple quadrupole (QqQ), the rest being methods based on IT and time-of-flight (TOF) analyzers. In the review article from Andreu and Picó ${ }^{1}$ on PRA in biota, 18 out of 24 papers reviewed dealt with the use of single quadrupole GC-MS.

Despite the wide existing applications, methods based on the use of single quadrupole instruments suffer from low sensitivity when working in the full scan mode. The sensitivity can be improved by working in the SIM mode, but the identification potential and the non-target/retrospective analysis capabilities are sacrificed. After development of the single quadrupole, the next step in the evolution of mass analyzers in pesticide residues analysis (without eliminating the use of the single quadrupole) was the increased use of ion trap mass spectrometers that allowed full spectra based methods to be developed with suitable sensitivity (similar to that obtained by a quadrupole in the SIM mode) in a single run. A number of papers have been published demonstrating the capability of ion trap analyzers for carrying out tandem MS experiments, improving sensitivity and selectivity, but losing the non-target capabilities. The fact that the MS/MS working mode of an ion trap is a product ion scan results in the co-elution of several analytes, or sample matrix components and notably reduces sensitivity and the number of points across the chromatographic peak. In the late 90s, the introduction of GC-TOF MS resulted in an improvement of full scan based methods and a step forward in non-target 
analysis. TOF MS is able to provide full spectrum acquisition data at high sensitivity. High-speed (HS) TOF MS, with a fast data acquisition rate (up to 500 spectra per second), is an excellent technique for GC $\times$ GC MS detection, for which the data acquisition speed is the most limiting factor. On the contrary, mainly coupled to 1D-GC and with lower data acquisition rates $(20-25 \mathrm{~Hz})$ and a narrower dynamic range, highresolution (HR) TOF MS provides sensitive full spectrum data with high mass accuracy, allowing the resolution of peaks from closely related interfering matrix components. In the last years, many papers can be found dealing with the determination of pesticides in food and environmental samples by GC $\times$ GC-(HS) TOF $\mathrm{MS}^{3,4}$ but much less related

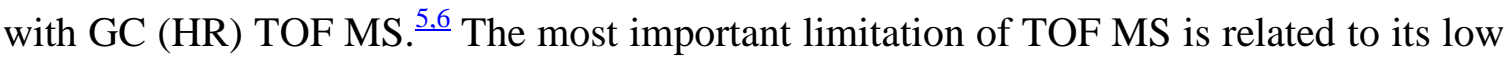
sensitivity which can make it troublesome to reach the required limits of detection (LOD) and, in the case of the HR TOF, also the peak saturation problems that occur in short dynamic ranges.

One of the major developments in the field of PRA has been the commercialization of liquid chromatography (LC) coupled to QqQ MS systems, which has benefited greatly from the high sensitivity and selectivity of tandem MS in the selected reaction monitoring (SRM) mode. Thus, QqQ has been the analyzer most used in LC-based methods in the 2006-2009 period, ${ }^{2}$ although this fact was not such evident for GC-based methods, which have suffered a notable delay in the wide acceptance of this analyzer in comparison to LC-MS/MS. In fact, LC-QqQ MS/MS started to be applied in PRA in the early 90's, while GC-QqQ MS/MS was applied around 15 years later. The first publications, between 2003 and 2005, reported the use of GC-QqQ MS/MS in food matrices like tobacco, oil, baby food or cucumber $\frac{7-10}{}$ and in human fat. ${ }^{11}$

Several papers have been published on the comparison of different analyzers in GCMS. Mezcua et al. $\frac{12}{}$ compared GC-Q MS and GC-IT MS(/MS) for the determination of insecticides in vegetables indicating that no significant differences were found in terms of sensitivity, although IT under MS/MS conditions was superior to GC-Q MS in the identification capability. Garrido et al. $\underline{13}$ made an interesting comparison between two MS/MS systems, QqQ and IT, concluding that intraday-precision was similar for both, but interday-precision was found to be worst in the case of QqQ. In contrast, better linearity ranges were achieved for QqQ together with lower matrix effects especially for 
dirty samples e.g. fat containing samples. Additionally, a larger number of compounds can be included in a single injection in QqQ (SRM mode), although regarding identification capabilities IT in the tandem MS mode gives more information for a better confirmation of positive findings due to the $\mathrm{MS}^{n}$ possibilities.

Several ionization techniques have been used in GC-MS over time, $\frac{14,15}{}$ electron ionization (EI) being the most popular and widely used. EI offers valuable information on the molecule structure (several fragment ions and, in some cases, molecular ions). It is a robust and universal ionization source that generates highly reproducible spectra that can be searched in available commercial spectral libraries for the identification of non-target compounds. However, EI generates highly extensive fragmentation. In some cases, it leads to mass spectra without abundant/intense characteristic peaks (e.g. molecular ion), and the sensitivity obtained can be poor. In those cases, alternative approaches, such as chemical ionization (CI), which can be applied in both the positive or negative mode allow mass spectra to be obtained with a predominant molecular ion peak and low fragmentation. CI has been applied in PRA, especially for the determination of organohalogenated pesticides due to its better sensitivity and selectivity for some of these compounds. $\frac{16,17}{}$ However, there is a low number of applications compared to EI, and it is not posible to carry out spectral library searching as it is not commercially available for CI.

A promising source is atmospheric pressure chemical ionization (APCI), which opens a new perspective in the development of GC-MS/MS methods. This source has been recently tested in PRA and offers very attractive features for compounds that suffer extensive fragmentation in the EI mode. $\frac{18,19}{}$

\section{GC-MS/MS applications in food analysis}

Nowadays, the control of pesticide residues in food commodities has become a requirement for compliance with the legislation, ensuring safety of the population and international and national trade. The determination of GC-amenable pesticides in food samples by using tandem MS with a QqQ analyzer has emerged in the last decade as a 
valuable approach, which allows higher selectivity and sensitivity and minimizes or even removes most chromatographic interferences. This section reviews the papers published in the last ten years related to the determination of pesticide residues in food samples by GC-QqQ MS/MS (Table 1). The most relevant aspects related to the studied pesticides, types of matrices, sample preparation procedures and analytical measurements are discussed in the present review.

The different physicochemical properties of the pesticide chemical classes increase the difficulty when developing a simultaneous analytical method for multiresidue analysis of food commodities. Thus, analytical methodologies based on GC-QqQ MS/MS for the determination of pesticides from the same family are quite common, e.g. for the determination of organochlorine (OC),$\stackrel{8,20,21}{ }$ organophosphorus (OP) pesticides, $\stackrel{22-24}{\text { or of }}$ pyrethroids $\frac{19,25}{}$ in food commodities. Some particular examples are the GC-QqQ MS/MS method developed by Le Faouder et al. ${ }^{26}$ just for fipronil, or by Peruga $e t a l$. for chlorothalonil. ${ }^{27}$ Other authors have included two families of pesticides, typically OC and OP. ${ }^{28-32}$ However, the majority of applications (around $70 \%$ ) deal with multiresidue methods for multiclass pesticides in food samples, $\frac{7,9,10,16-18,33-58}{1}$ the most adequate strategy available for monitoring purposes that minimizes time, costs, reagents, labors and hazards in order to obtain rapid analytical results in response to urgent demands. Moreover, most multiclass methods published include more than one hundred pesticides in their target list, among insecticides, herbicides, acaricides, fungicides, etc. ${ }^{10,33,35-}$ $\underline{38,40,41,43,44,46-52,57}$ In this respect, remarkable papers are those published by Okihashi et $a l . \frac{35}{}$ and by Banerjee et al., ${ }^{57}$ who have developed analytical methodology for the determination of up to 260 and 349 pesticides in fruits and vegetables, respectively. 


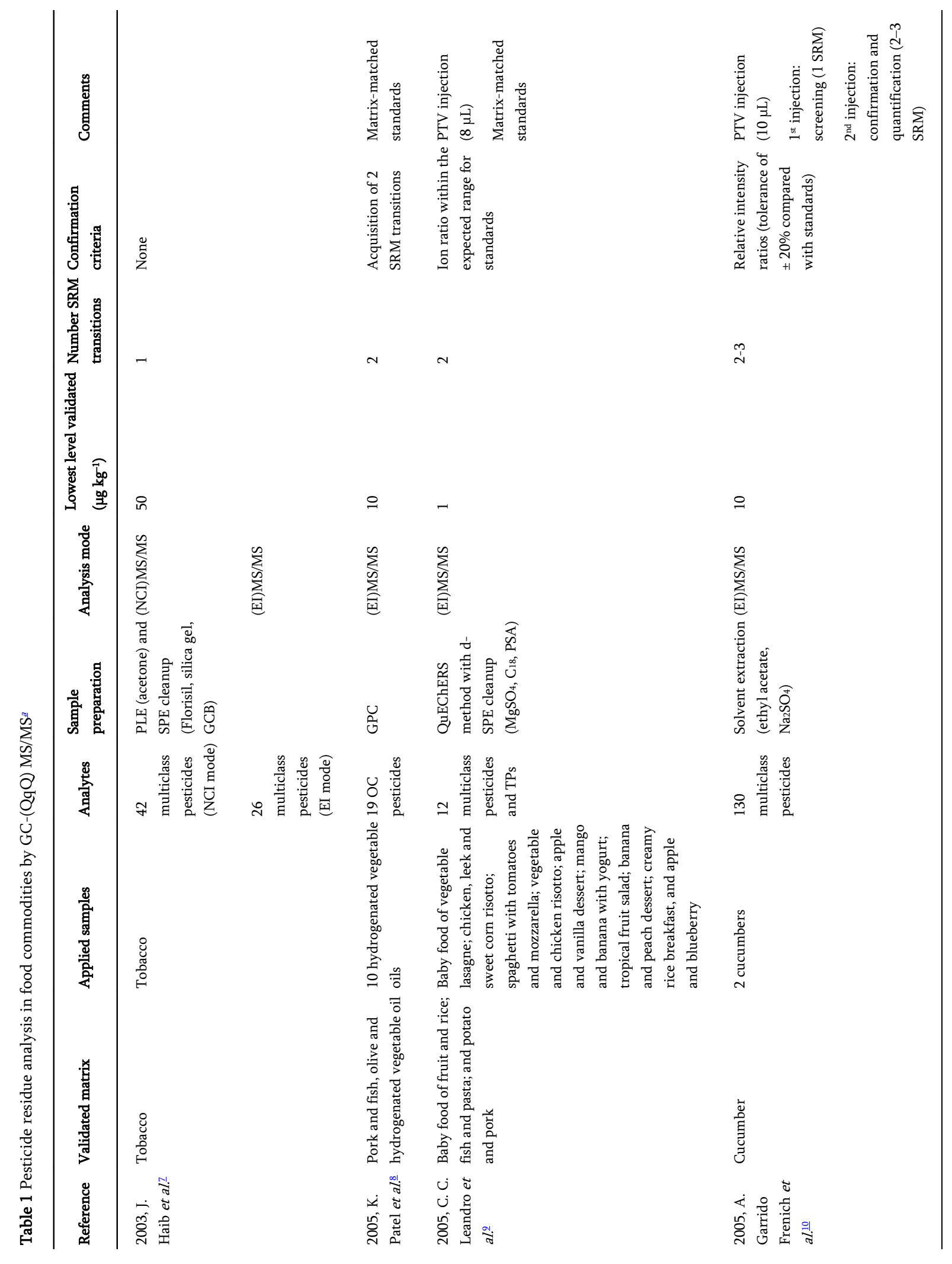




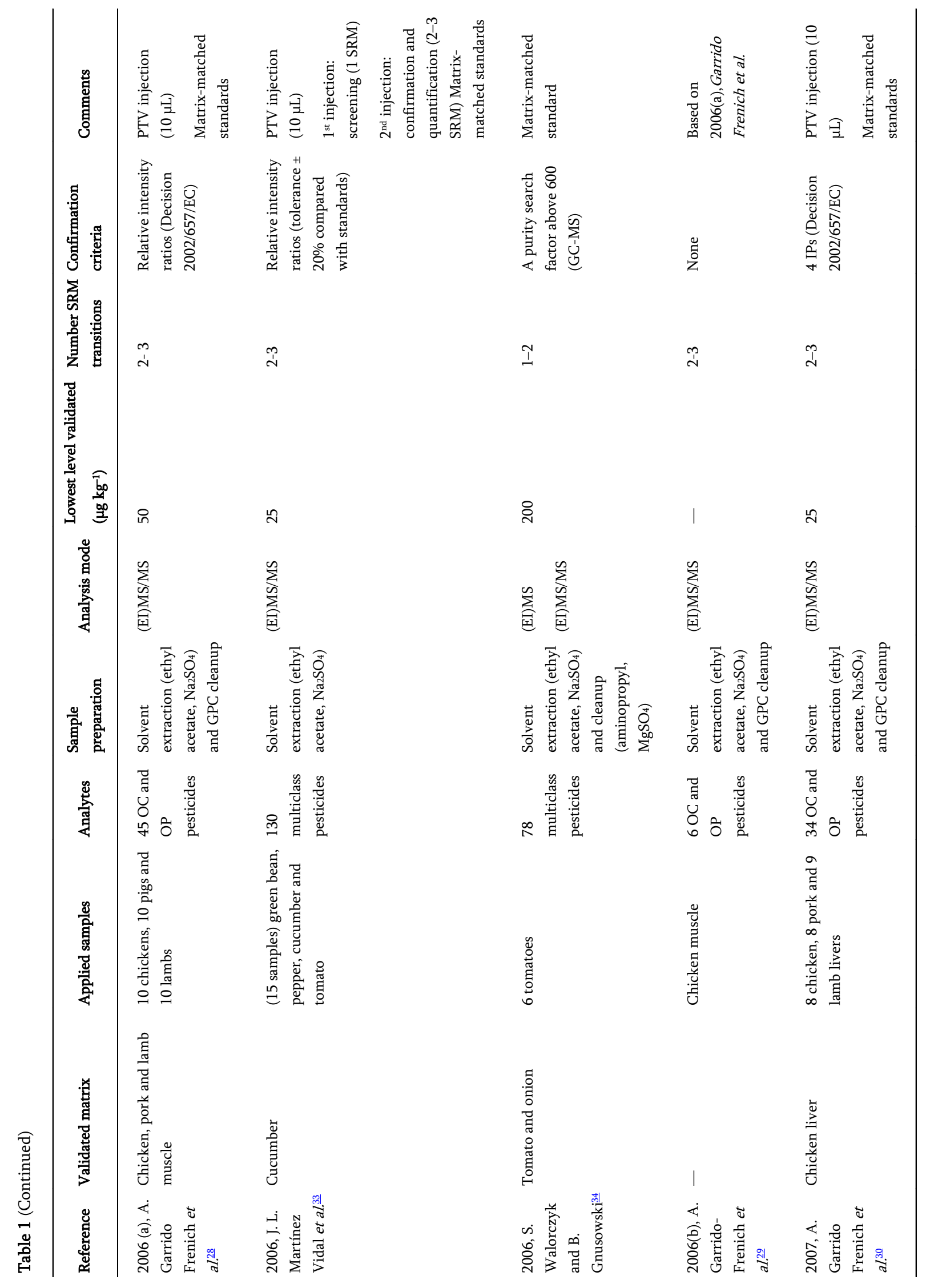




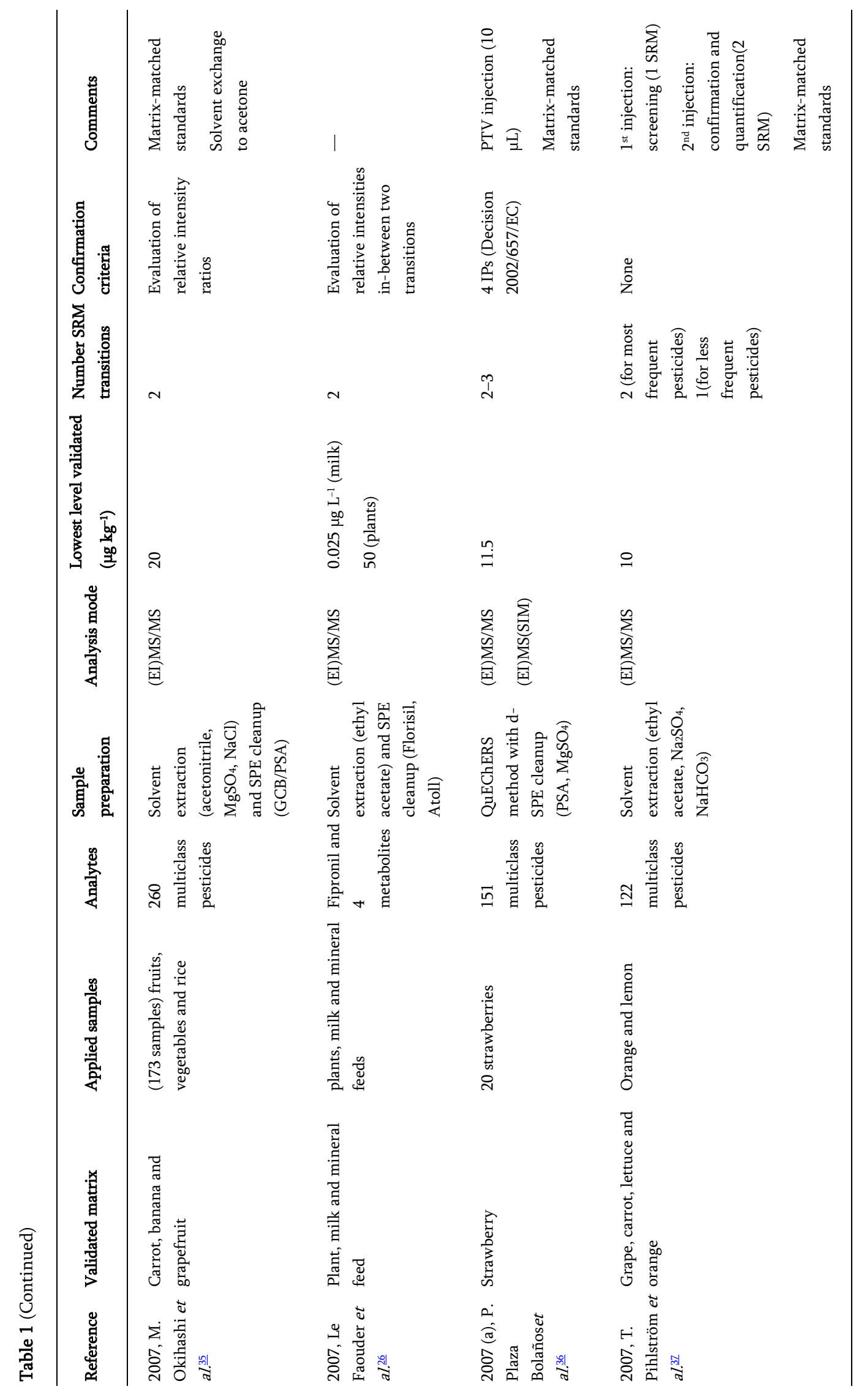




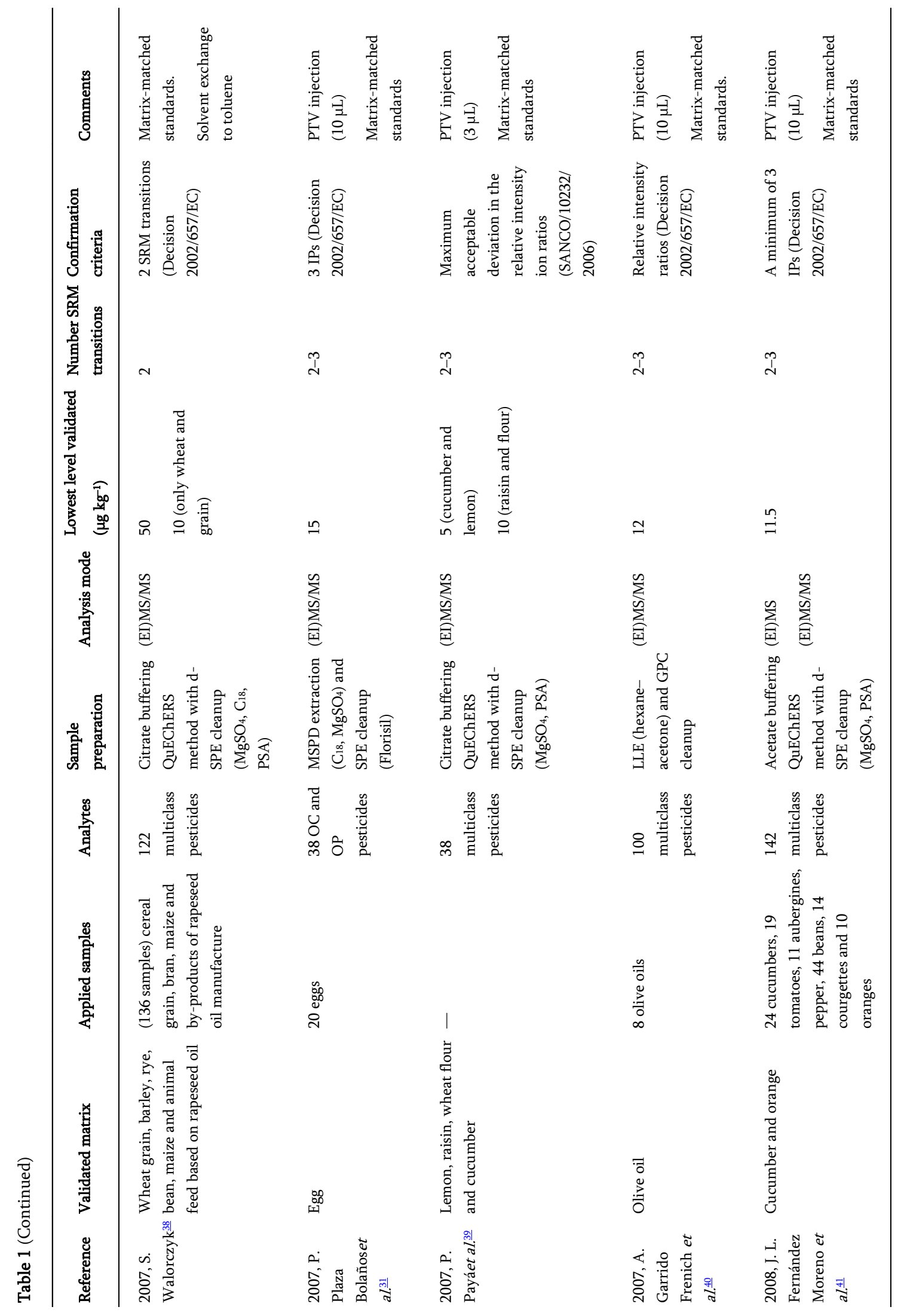




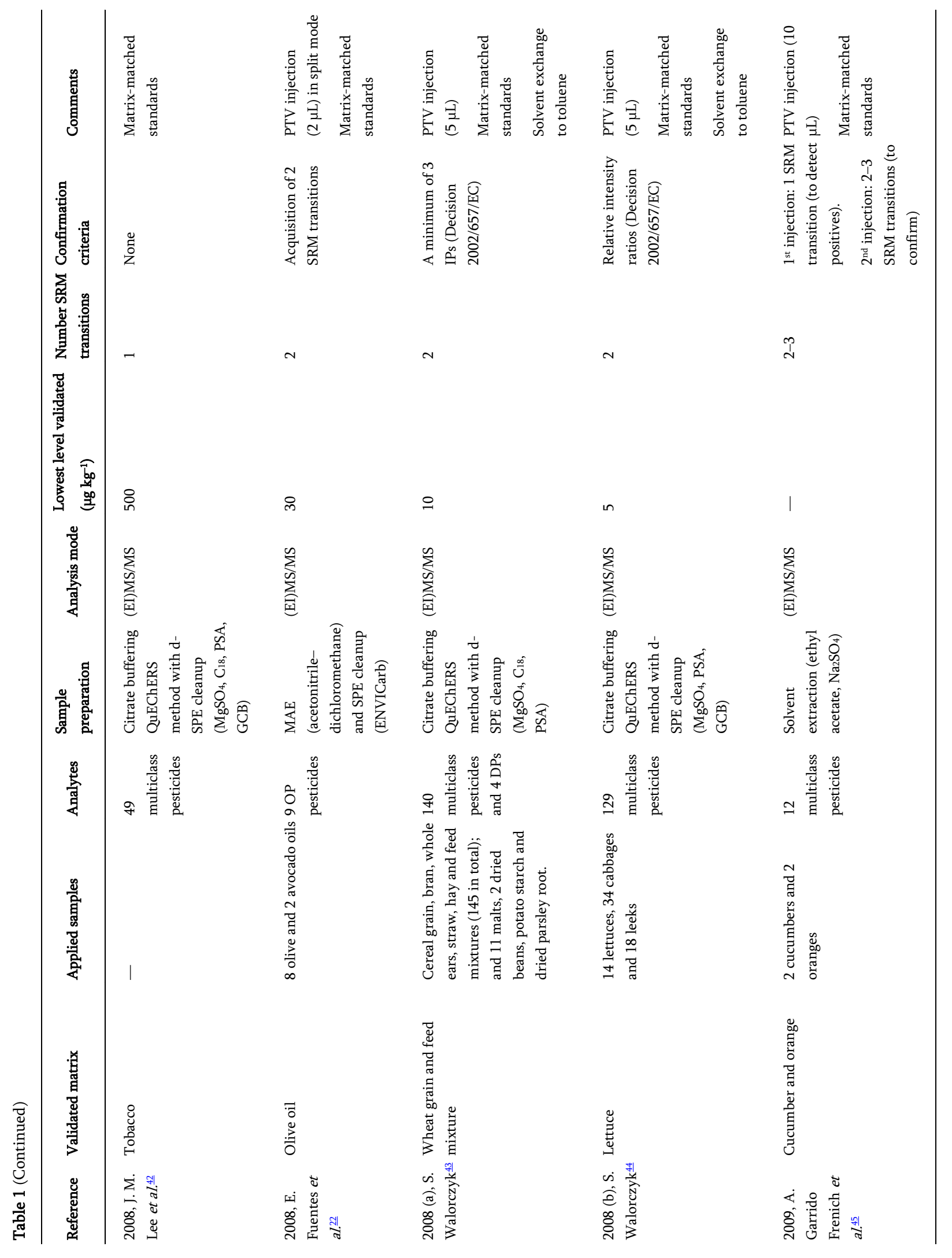




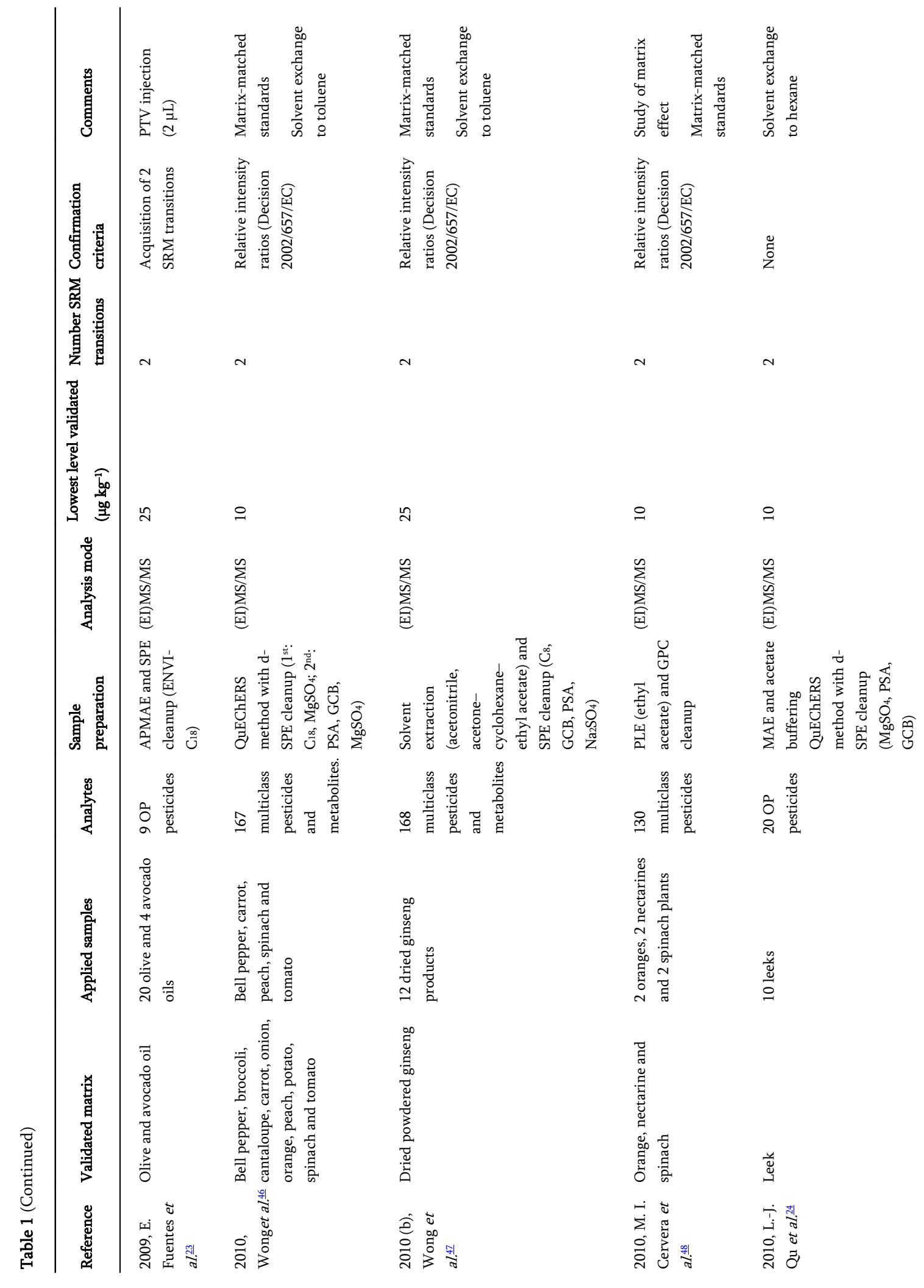




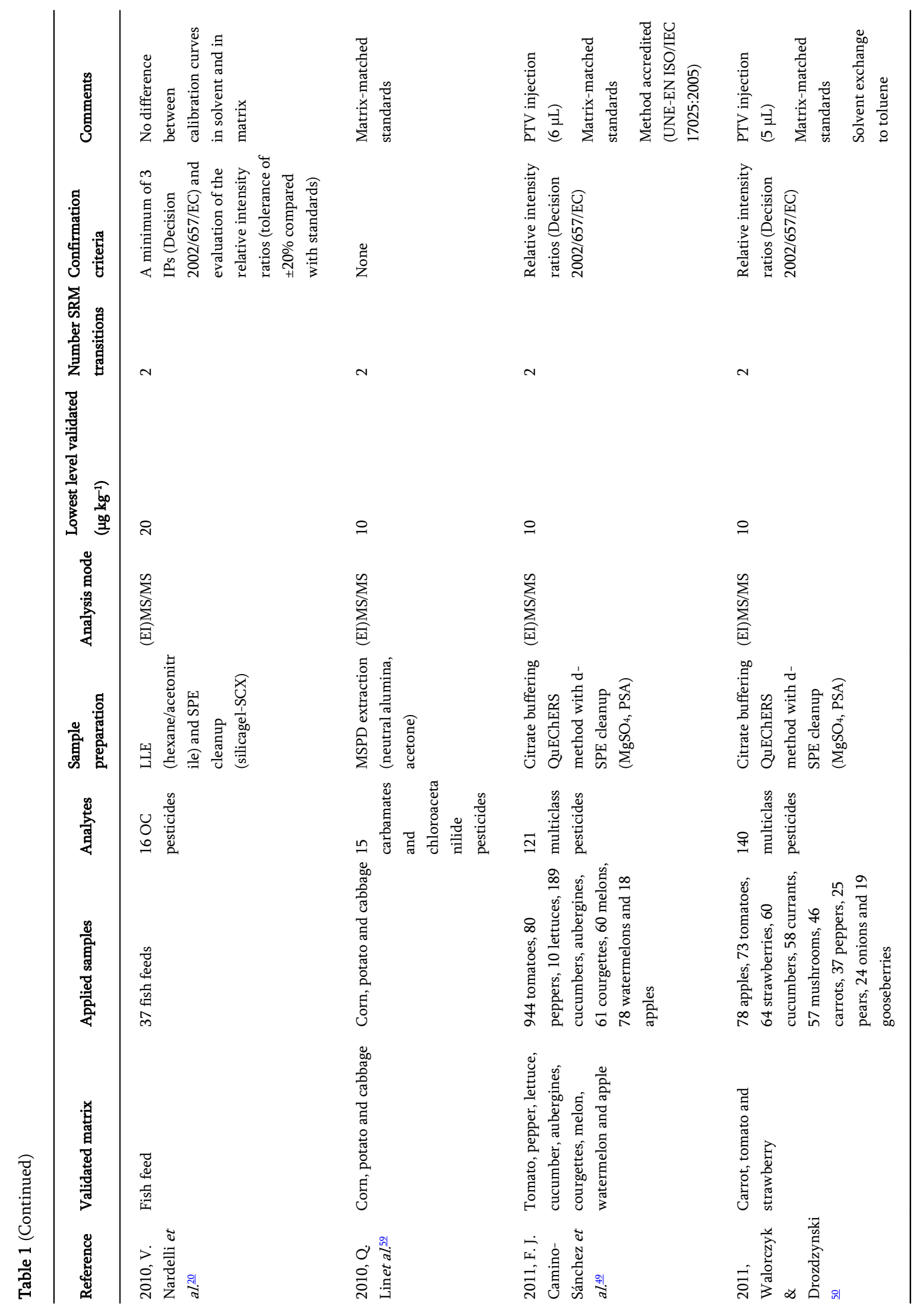




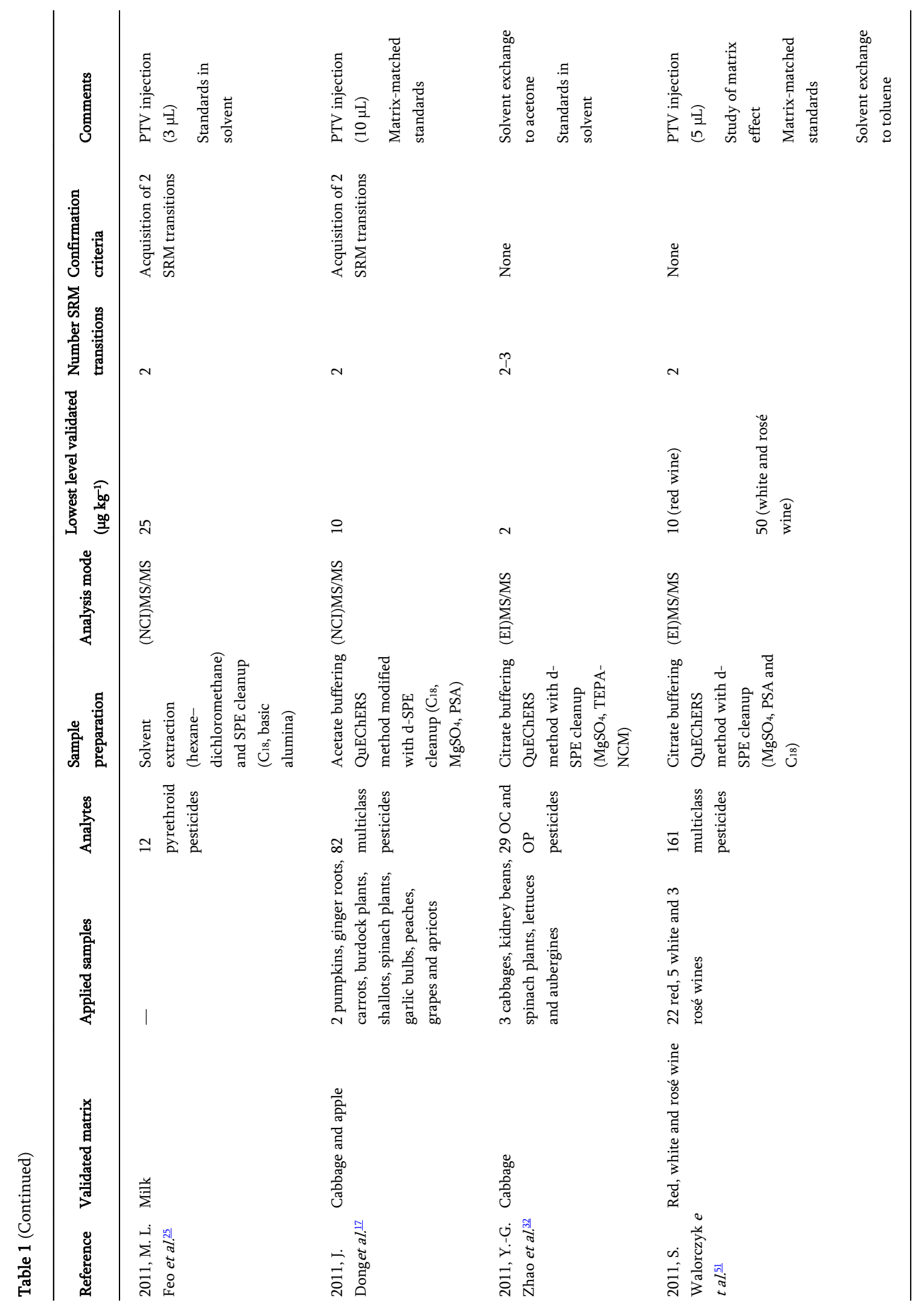




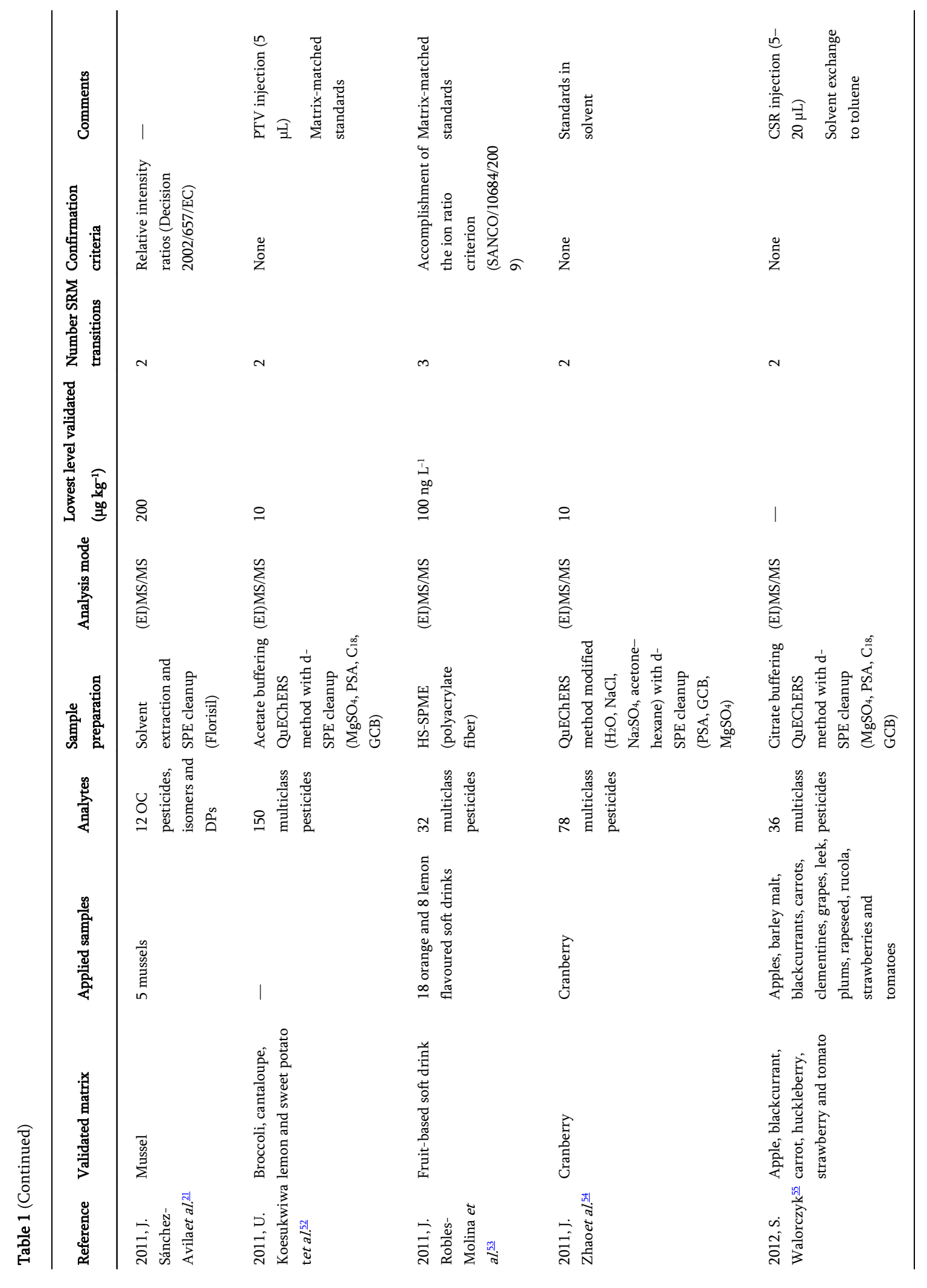




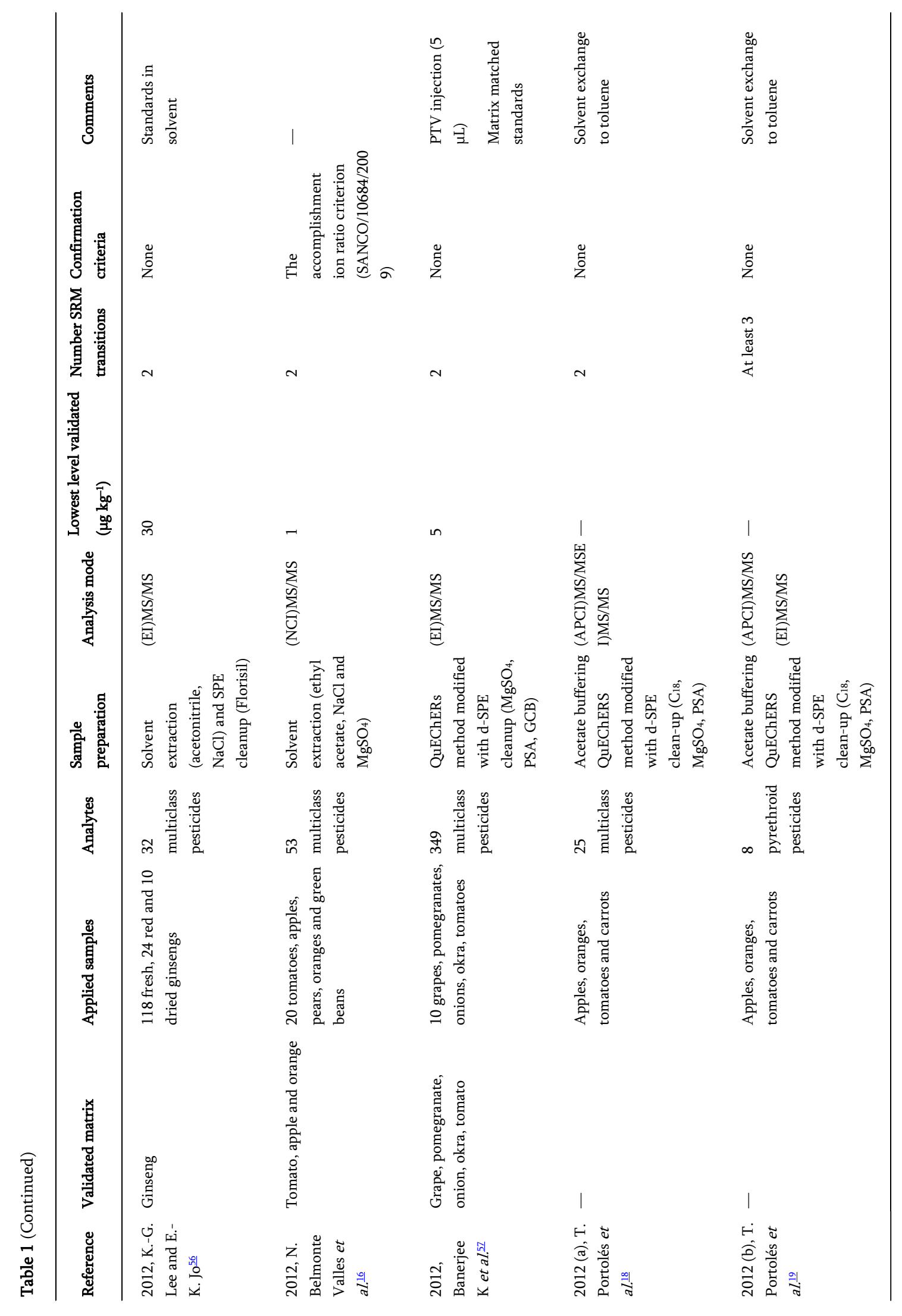




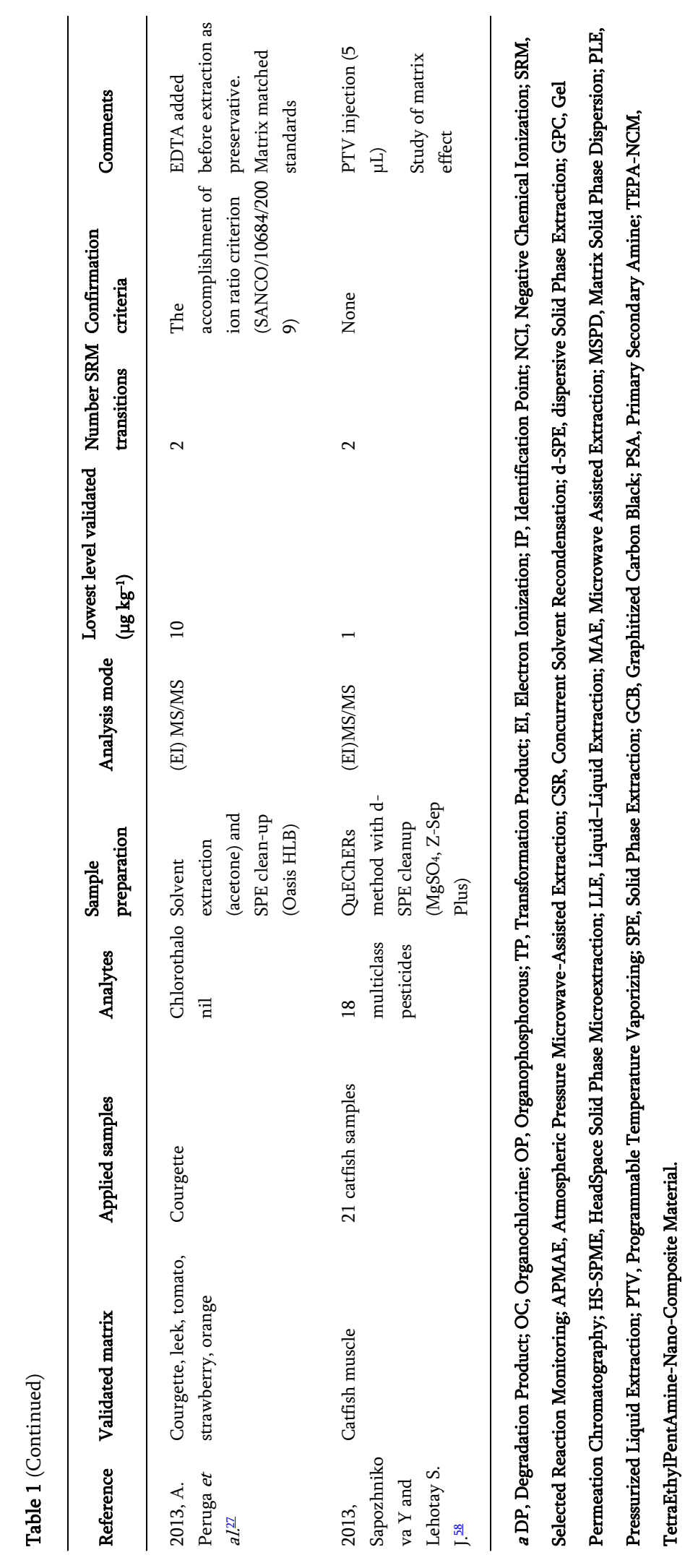


In PRA, the term Food includes a wide range of treated products, fruits, vegetables, grains and other commodities. Even after being washed, stored, processed and prepared, some pesticide residues may remain in both fresh products and processed foods. From the overview of the applications shown in Table 1, it can be seen that fruits and vegetables are the most frequent samples analyzed. $\frac{10,16-19,24,27,32-37,39,41,44,46,48-50,52,54,55,57,59}{\text { Other }}$ matrices analyzed are oils and fats,,$\frac{8,22,23,38,40}{}$ cereals,,$\frac{38,39,43,59}{}$ muscles and livers,, $28-$

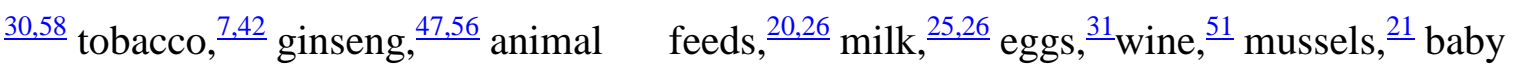
food $^{9}$ and fruit-based soft drinks..$^{53}$

GC-MS/MS methods for pesticide residues include the extraction of the analytes from the matrix, appropriate cleanup of the raw extracts and final measurement. The most used approach for extraction of pesticides from food samples is nowadays the QuEChERS procedure (Quick, Easy, Cheap, Effective, Rugged and Safe), which has been widely reported in the literature. ${ }^{60}$ Some variations of the original method have led to two modified methods: the acetate buffered method (AOAC official Method 2007.1) $\frac{61}{1}$ and the

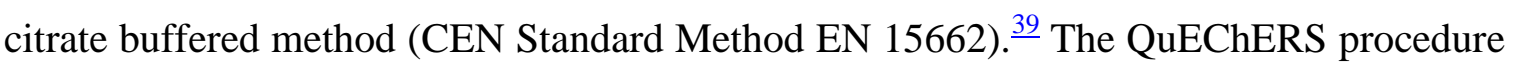
in combination with GC-QqQ MS/MS is one of the preferred approaches at present for residue determination of GC-amenable pesticides. Among the methods reported, it can be mentioned that those of Leandro et al., ${ }^{9}$ Plaza Bolaños et al. ${ }^{\frac{36}{6}}$ and Wong et al.,${ }^{46}$ used the original method without modifications. In contrast, other authors obtained good results using the acetate buffered version, $\frac{17-19,41,52}{}$ although citrate modification seems to be more used in this field. In fact, the citrate buffered version has become the most applied extraction method in pesticide residue analysis in food by GC-QqQ MS/MS. 51,55 In these methods, a subsequent cleanup step is applied based on dispersive-solid phase extraction (d-SPE) using different sorbents, such as primary secondary amine (PSA), $\mathrm{C}_{18}$, Z-Sep Plus and/or graphitized carbon black (GCB), depending on the complexity of the matrix.

Obviously, other sample preparation procedures have also been applied. Some authors performed a simple extraction with solvents such as ethyl acetate, $\underline{10,16,26,28-}$ $\underline{30,33,34,37.45 .48}$ acetone,,$\frac{7,27}{}$ acetonitrile,$\stackrel{35.47 .56}{ }$ or mixtures hexane-acetone, $\stackrel{40}{~ h e x a n e-}$ acetonitrile ${ }^{\underline{20}}$ or hexane-dichloromethane. ${ }^{25}$ Most of the reported procedures require the application of an additional cleanup step to remove interferences and also to improve 


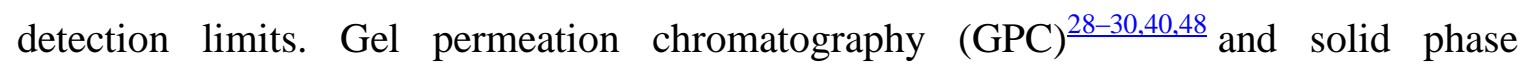
extraction (SPE) $)^{7,20-23,25,26,31,35,47,56}$ have been commonly applied for this purpose. As an exception, Robles-Molina et al. $\frac{53}{}$ proposed a method consisting of a solventless sample treatment procedure based on headspace solid-phase microextraction (HS-SPME) for the determination of target pesticides in fruit-based soft drinks.

After sample preparation, the final extract is commonly injected into the inlet system with a classical split/splitless injector. In some cases, the final extracting solvent is not appropriate for injection into the split/splitless system, due to the high volume-expansion coefficient during vaporization. Solvent exchange prior to chromatographic injection to an adequate solvent such as toluene, $\frac{18,19,38,43,44,46,47,50,51,55}{\text { acetone }} \frac{32,35}{}$ or hexane $\mathrm{e}^{\underline{24}}$ could be a good choice to solve this problem. Another option is the use of programmable temperature vaporizing (PTV), which is also employed to improve the limits of detection in PRA as it allows the injection sample volumes higher than the typical ones $(1-2 \mu \mathrm{L})$ in a split/splitless injector. ${ }^{9,10,17,25,28,30,31,33,36,39-41,43-45,49-52,57,58,62}$ Apart from PTV, other large volume injection (LVI) systems are on-column injection or concurrent solvent recondensation $(\mathrm{CRS})$ injection. $\underline{55}$

Regarding to the GC-QqQ MS/MS measurement (Table 1), EI is the most used ionization mode for the determination of pesticides in food, although the use of negative CI (NCI) has also been reported. $\frac{7,16,17,25}{}$ For some compounds, with highly electronegative elements, such as halogen, oxygen, etc., the use of the NCI mode usually provides better sensitivity and selectivity. The use of the QqQ allows selected reaction monitoring (SRM) to be applied, one of the most selective and sensitive approaches for simultaneous quantification and confirmation in PRA, when adequate precursor and product ions are selected. In this way, most matrix interferences are minimized, or even eliminated, improving the selectivity and the sensitivity, and reaching low detections limits due to the lower chemical noise in the chromatograms.

In general, the criteria used for confirmation/identification of positive samples are not treated in detail in most of the papers published. Some authors do not mention this issue, and only acquire one SRM transition for each analyte without mentioning any confirmation criteria. $\frac{7,37,42}{}$ Other authors propose the acquisition of two SRM transitions, but do not mention which criteria are applied to consider positive 
confirmation. $\frac{18,24,29,32,37,51,52,54-59}{}$ Some authors $\frac{8,17,22,23,25}{}$ have used the mere presence of a second SRM transition as confirmation criteria for positives in the samples. However, the European Commission Decision 2002/657/EC ${ }^{\underline{63}}$ implements the concept of identification points (IPs). In the case of MS/MS determination, 1 identification point is earned from a precursor ion, and 1.5 identification points are earned from a resulting product ion. For the unequivocal confirmation of the identity of compounds at least 3 and 4 identification points are required for legal and banned substances, respectively. ${ }^{43}$ This has been applied

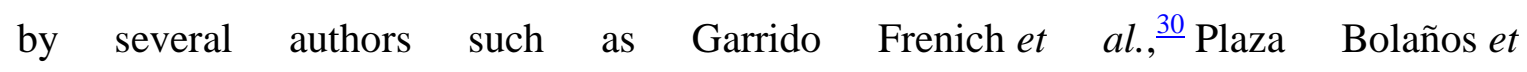
al., $\stackrel{31,36}{ }$ Walorcyzk $\frac{38,43}{}$ and Fernández Moreno et al..$^{41}$ in their work. Nowadays, the most widely accepted approach is based on the presence of chromatographic peaks at the two (or three ${ }^{53}$ ) transitions acquired, together with agreement of the $R_{\mathrm{t}}$ and the evaluation of the intensity ratio between the quantification $(Q)$ and the confirmation $(q)$ transition, and comparison with those of the reference standard within the maximum tolerances

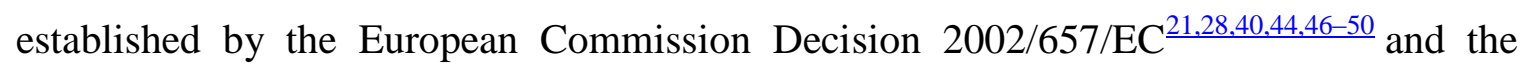
$\mathrm{SANCO}^{64}$ guidelines. $\frac{16,27,39,53}{}$

Modern QqQ instruments allow the simultaneous acquisition of two or more transitions in just a single GC analysis. However, some publications

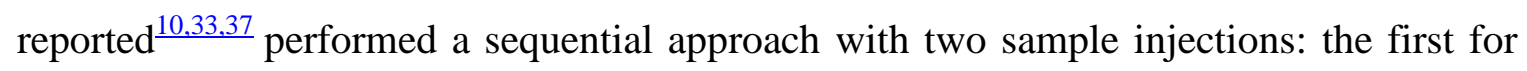
rapid screening, with acquisition of only one transition, and the second for the confirmation and quantification of the compounds previously detected in positive samples, with acquisition of 2 or 3 transitions. As a particular example, Fuentes et $a l .{ }^{22,23}$ determined OP pesticides in olive oil by GC with flame photometric detection (GC-FPD) and the identity of residues in positive samples was confirmed through GCQqQ MS/MS analysis by acquiring two transitions.

Once the identity of the analyte in the sample has been confirmed, quantification of pesticide residues is normally the next objective. Different approaches have been reported for quantification of pesticide residues in food, commonly considered as a complex matrix. Matrix-matched standards calibration is a good option for quantification of analytes affected by matrix effects, and it has been widely applied in PRA of food commodities..$^{8,9,17,22,27-31,33-53,57,59}$ Cervera et al. ${ }^{48}$ studied the matrix effect of several food matrices (orange, nectarine, spinach, raisin, paprika, cabbage, pear, rice, legume and 
gherkin) comparing the response of reference standards prepared in solvent with the response of matrix-matched standards. Most of the pesticides showed an evident signal enhancement in the presence of matrix, and matrix-matched calibration using relative responses to an internal standard was required for the correct quantification of compounds. On the contrary, Nardelli et $a l .{ }^{20}$ performed the quantification of OC pesticides in fish feed by using standard solutions in solvent and in the matrix as calibration curves, no differences were observed between the results obtained using the different sets of standards. Other applications have been reported in the literature in which standard solutions in solvent were used for analysis of different matrices such as cabbage, $, \frac{32}{m i l k}, \frac{25}{}$ mussel $^{21}$ and ginseng. $\frac{56}{}$ As occurs with other analyzers used in GC-MS, the triple quadrupole analyser, even working in the SRM mode, is affected by coextracted matrix components that may lead to an enhancement of the chromatographic signal or to a reduction of the analyte response in comparison to the signal in pure solvent, as these effects are normally a consequence of problems coming from the GC system. Thus, when coextracted matrix components compete with the target pesticides to access the active sites of the GC system and/or when they are protected from decomposition in the hot injector, a matrix-induced response enhancement is observed. Conversely, accumulation of non-volatile coextracted matrix components in the GC system helps to generate new active sites, and matrix-induced response diminishment occurs. $\underline{45}$

Sensitivity is an important parameter to measure the potential of a method in PRA. LOD and LOQ are usually calculated for this purpose, although their estimation is a controversial issue, due to the different ways of calculation. This makes it troublesome to perform a realistic comparison of the values reported in the literature. In order to compare the sensitivity of the applications reviewed, the lowest concentration level validated was used as an indicator of the sensitivity of GC-MS/MS methods. Most of the publications (Table 1) used a lowest level validated in the range of $5-10 \mu \mathrm{g} \mathrm{kg} \mathrm{g}^{-1} . \underline{8,10,17,24,27,37-}$ $\underline{39,43,44,46,48-52,54,57,59}$ The use of a large injection volume resulted in an increase in the sensitivity, and lowered the method validation concentration down to $1 \mu \mathrm{g} \mathrm{kg}^{-1}$. Thus, Leandro et al., ${ }^{9}$ Belmonte Valles et al. ${ }^{16}$ and Sapozhnikova and Lehotay ${ }^{58}$ validated their procedure at the lowest level of $1 \mu \mathrm{g} \mathrm{kg}^{-1}$ in baby foods, fruits and vegetables, or in catfish muscle, using large volume injections of 8,2 and $5 \mu \mathrm{L}$, respectively. 


\section{GC-MS/MS applications in the analysis of environmental samples}

The extensive use of pesticides in agriculture and their industrial applications in the last decades, together with the persistence of some of these compounds, has led to their wide presence in the different compartments of the environment. Consequently, there is a need to know the concentration of these contaminants in the aquatic environment, although they normally are found at the $\mu \mathrm{g} \mathrm{L}^{-1}$ level or below. To this aim, strict regulations and environmental monitoring programs have to be adopted to accurately determine the concentration levels of pesticides. GC-QqQ MS/MS is an attractive technique with strong potential in the determination of low levels of pesticides in environmental samples, as occurs in food analysis.

The number of papers published until now related to environmental applications of GC-QqQ MS/MS in pesticide residue analysis is not as large as for food (Table 2). Only eighteen publications have been found and among them eleven developed analytical methodology for the determination of multiclass pesticides. $\frac{65-75}{}$ Only two articles deal with around hundred target analytes: Barco-Bonilla et al. $\frac{67}{}$ included 139 analytes in waste water samples analysis and Martínez Vidal et al. ${ }^{69}$ included 98 pesticides in soil analysis. It is noteworthy that the first publication dealing with the use of GC-QqQ MS/MS for pesticides together with other organic contaminants in environmental samples dated $2007, \frac{65}{}$ which illustrates the novelty and the recent use of this technique. 


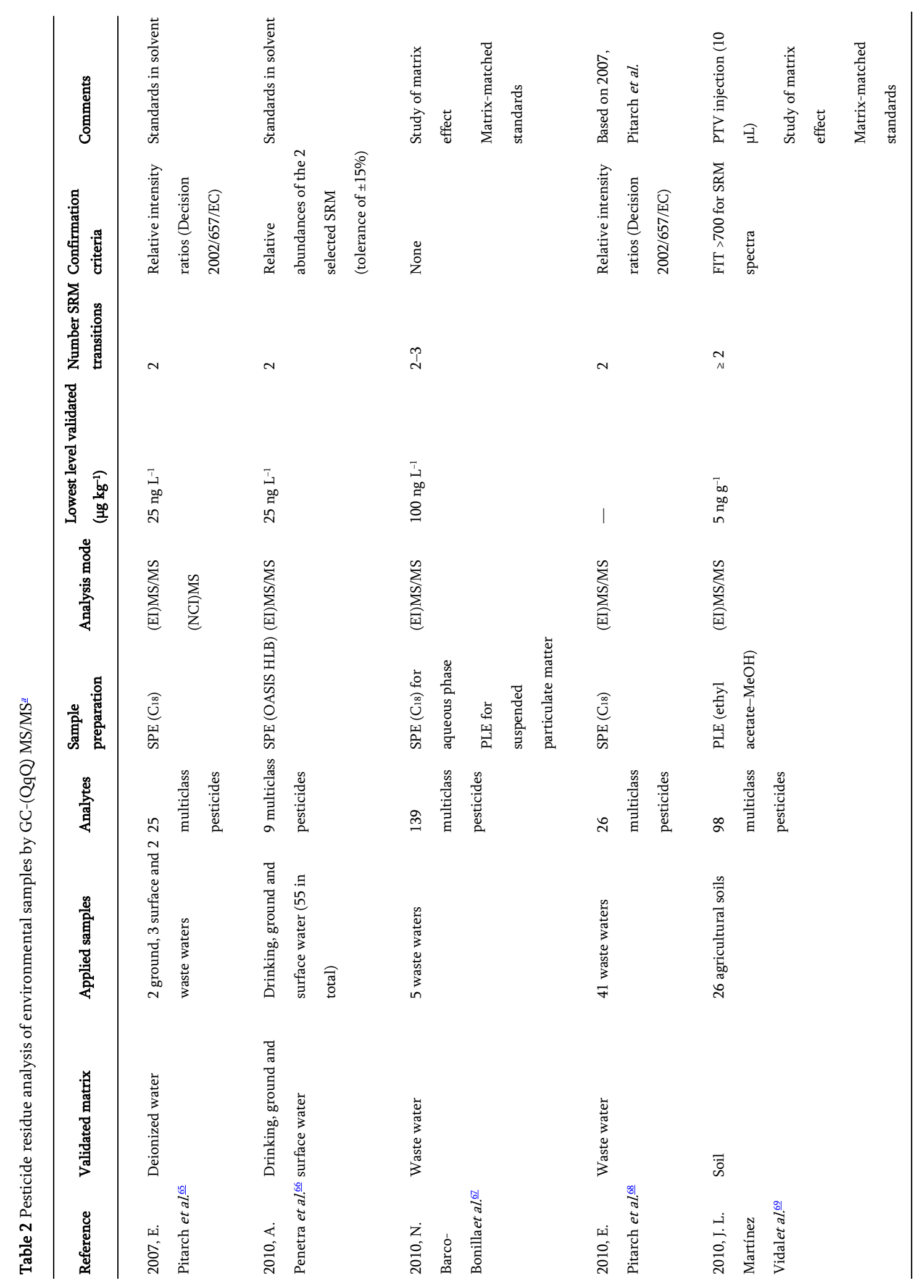




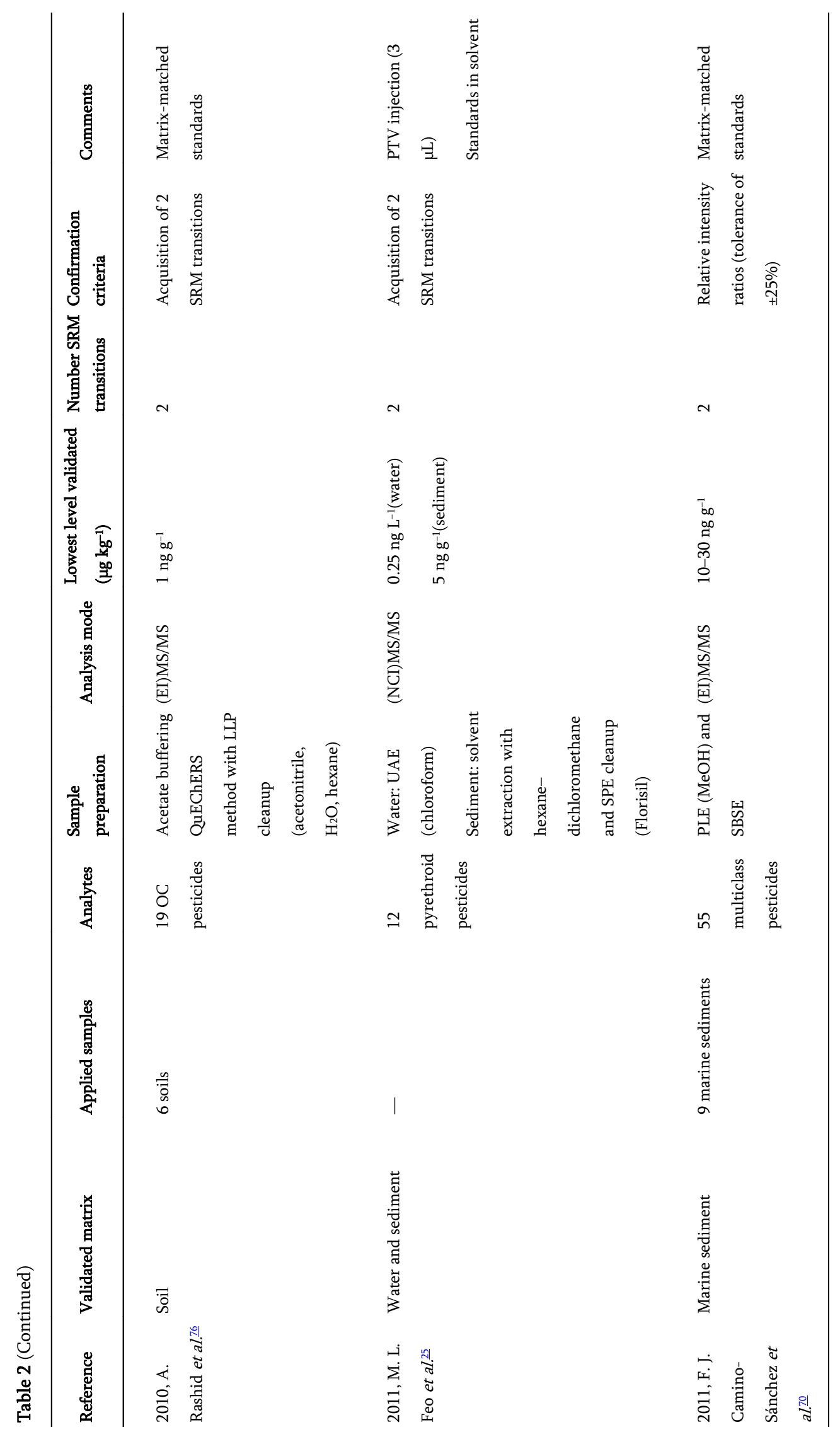




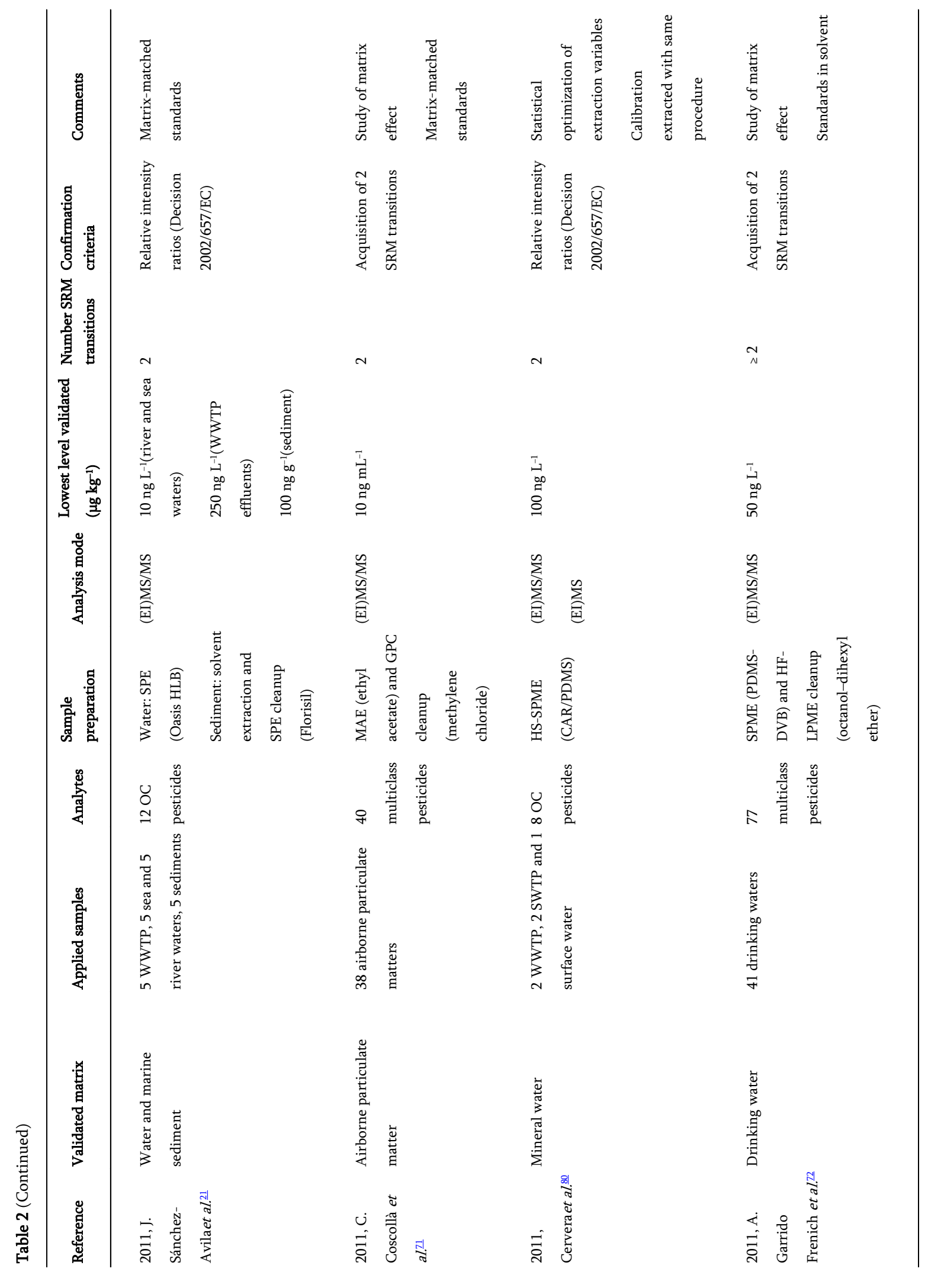




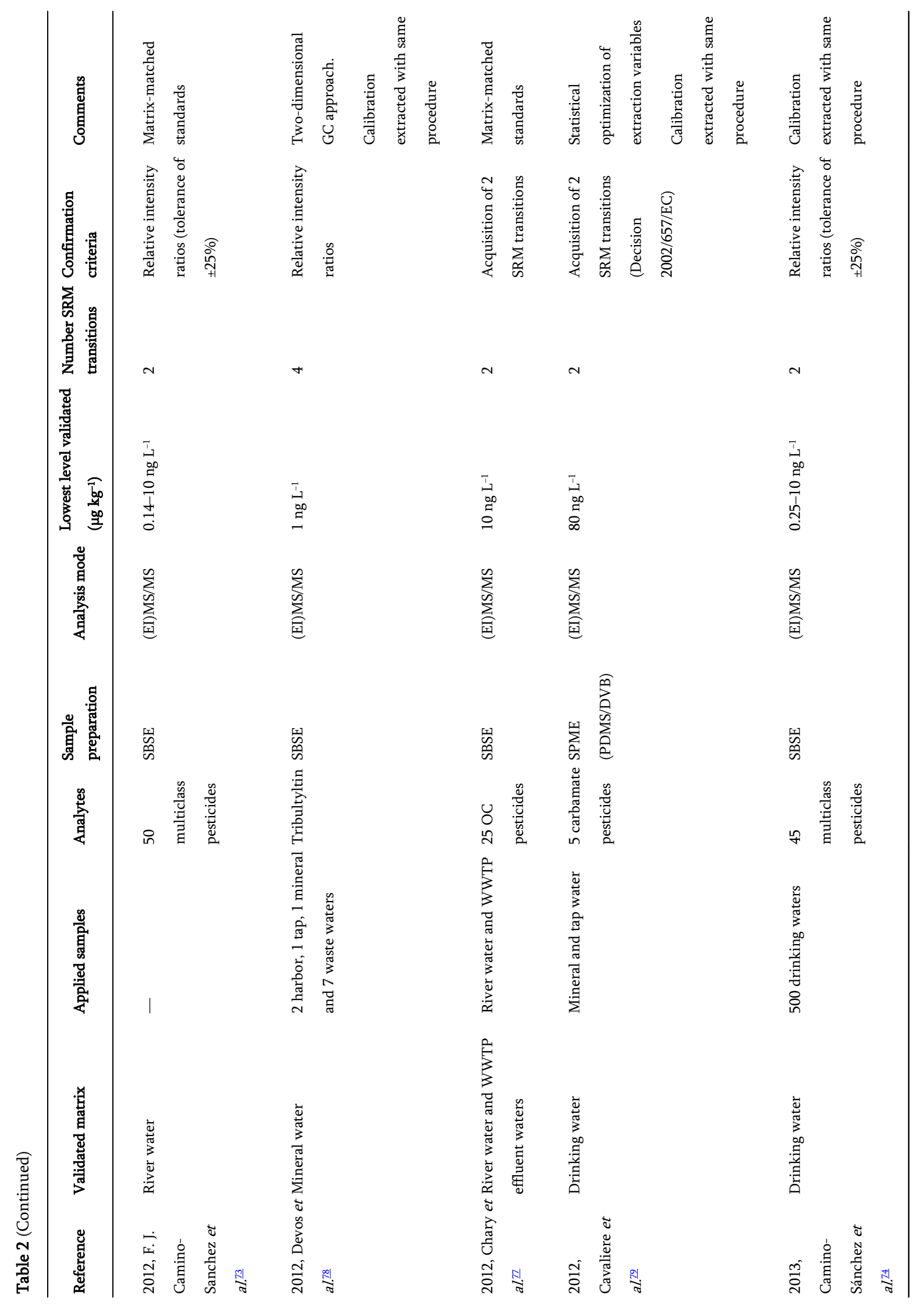




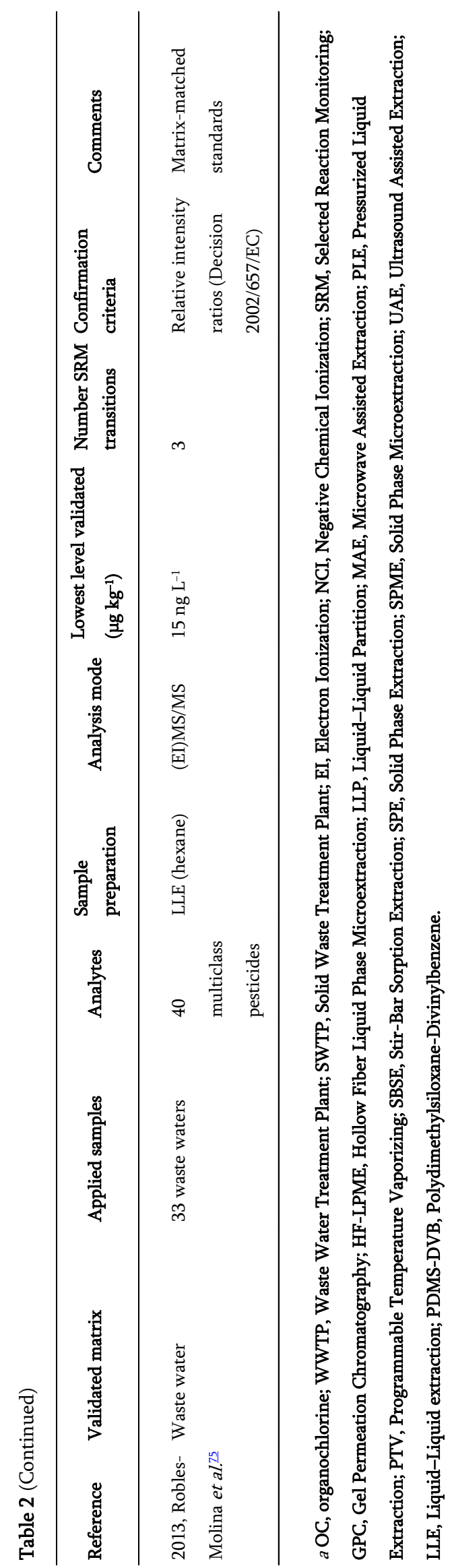


Depending on the complexity of the matrix, different techniques have been used to extract the analytes. For example, soil generally requires the use of stronger techniques capable of extracting potentially bound residues. Only two applications dealt with soil analysis: Martínez Vidal et al. $\stackrel{69}{ }$ who used a pressurized liquid extraction (PLE) technique with a mixture of ethyl acetate and methanol for investigation of multiclass pesticides, and Rashid et al. $\frac{76}{}$ who developed a methodology based on acetate buffering QuEChERS with a posterior liquid-liquid partition (LLP) cleanup for the determination of OC pesticides. In the case of sediments, analytical methodologies are based on extraction with different solvents followed by an additional cleanup or concentration step using $\mathrm{SPE}^{21,25}$ or stir-bar sorption extraction (SBSE)..$^{70}$ Airborne particulate matter has been another environmental matrix analyzed by GC-QqQ MS/MS, $\stackrel{71}{-}$ using microwave-assisted extraction (MAE) followed by GPC as a cleanup step. As shown in Table 2, water has been the most common matrix studied in the environmental field. SPE using different sorbents has been the technique of choice by most authors. ${ }^{21,65-68}$ Some exceptions applied to pyrethroids pesticides that were extracted using ultrasound-assisted emulsification extraction (UAEE) with chloroform, $\frac{25}{2}$ and SBSE which was used for the analysis of pesticides in river water ${ }^{73,77}$ or in drinking water. ${ }^{74,78}$ Garrido Frenich et al..$^{72}$ compared both SPME and hollow fiber liquid phase microextraction (HF-LPME) for the extraction of pesticides in drinking water, concluding that SPME and GC-MS/MS offered the best compromise in terms of quality, speed and reliability.

After extraction (and occasionally cleanup), GC-amenable pesticides described in Table 2 were determined by GC-QqQ MS/MS in the SRM mode. In all the publications, at least two transitions were acquired and most used the EI mode. Feo et $a l . \underline{25}$ concluded that the best selectivity and sensitivity for the determination of pyrethroids in water and sediment was obtained by using GC-MS/MS in the NCI mode. Pitarch et al. ${ }^{65}$ developed the first GC-MS/MS methodology for priority organic pollutants in water, including several pesticides. Although the EI mode was used for the general method, a supplementary methodology based on GC-(NCI) MS using the selected ion recording (SIR) mode was proposed for quantification and confirmation of OC pesticides as it allowed notable sensitivity improvement for these compounds. As regards confirmation identity, it is based on the presence of at least two SRM transitions $\frac{25,71,72,76,77,79}{}$ and $R_{\mathrm{t}}$ agreement, although several authors also took into account the experimental relative intensity ratio of the sample and the theoretical ratio of the 
reference standard, using the maximum deviations established in the European

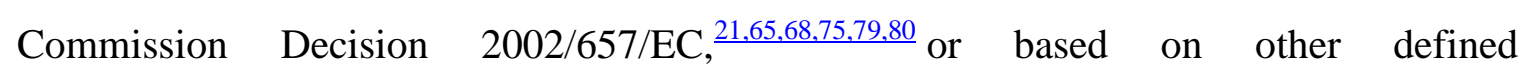
tolerances. $\underline{66,70,73,74,78}$

Quantification for water samples has been mostly based on calibration in solvent. ${ }^{25,65,66,68,72}$ For more complex matrices, matrix-matched calibration provided better results, as in the case of soil samples, $\frac{69,76}{}$ marine sediments $\frac{21,70}{}$ or even airborne particulate matter. . $^{1}$

The GC-MS/MS methods applied in the analysis of environmental samples presented excellent sensitivity. In the case of soils and sediments, the lowest level validated was as low as $1 \mathrm{ng} \mathrm{g}^{-1} \underline{16}$ or $5 \mathrm{ng} \mathrm{g}^{-1} \cdot \underline{25,69}$ The purity of the air was evaluated by analyzing the airborne particulate matter performing the validation at the lowest level of $10 \mathrm{ng}$ $\mathrm{mL}^{-1} \cdot \underline{11}$ As regards water samples, the lowest level validated is reported to be $0.14 \mathrm{ng}$ $\mathrm{L}^{-1}$ for a variety of priority organic pollutants. ${ }^{73}$ As expected, the lowest level validated in waste water was much higher, as a consequence of the higher matrix complexity. $\frac{67,75,77}{}$

\section{Trends and perspectives}

After its first use for PRA around 10 years ago, GC-QqQ MS/MS has been consolidated in most laboratories. This technique has the degree of robustness required to be widely applied at present, and the improvements offered as regards method sensitivity and selectivity are widely recognized. The determination of pesticide residues by GC-MS is commonly based on the use of relatively long capillary columns $(25-30 \mathrm{~m})$ with internal diameters of $0.25-0.32 \mathrm{~mm}$ by using typically low polarity stationary phases (from $100 \%$ methyl silicone to 5\% phenyl methyl silicone in most cases) leading to chromatograms of tenths of minutes. Fast GC coupled to MS has been shown to be an interesting alternative that, through different instrumental approaches, allows increased sample throughput by reducing the analysis time. ${ }^{81,82}$ In this way, low-pressure GC-QqQ MS/MS has been applied to pesticide residue analysis, with an important increase in sensitivity, shorter run time, higher sample loading and increased ruggedness. $\frac{81,83}{}$ Another approach is based on the use of narrower columns with internal diameters of $0.1 \mathrm{~mm}$ i.d. combined with fast column temperature programming, resulting in an increase in sensitivity, a reduction in the analysis time and peak width and thus an increase in resolution, thereby making it 
feasible to determine even more than one hundred pesticides in analysis times lower than 10 minutes. $\underline{.82,84}$

The use of APCI as an ionization source for GC-MS methods, is a major advance that will greatly improve pesticide residue determination (and other GC amenable compounds) due to its soft ionization behaviour in comparison with that obtained by EI. Portolés et al. $\frac{18,19}{}$ have demonstrated the capabilities of this soft ionization source for producing spectra with much lower fragmentation than that obtained by EI, where the molecular ion is commonly absent (or with low abundance). In these cases, when using EI it is necessary to select a fragment ion as a precursor ion in the MS/MS method and, consequently, not only the sensitivity but also the specificity of the method can be affected. With APCI, $\mathrm{M}^{+}$or $[\mathrm{M}+\mathrm{H}]^{+}$is the base peak of the spectra in most cases. Under these conditions, precursor-ion selection would no longer require a compromise between selectivity and sensitivity, allowing more specific MS/MS experiments. This approach has not yet been exploited in pesticide residues analysis but it will surely be a major advance in this field in the near future.

A combination of GC-MS/MS and LC-MS/MS, both with a triple quadrupole analyser, is one of the most current powerful approaches in PRA. They are complementary techniques that allow the determination of pesticides and metabolites within the whole range of physico-chemical properties, such as volatility, polarity and thermal stability. The combined use of both techniques allows the monitoring of hundreds of compounds that are GC or LC amenable. The present trend in multiresidue analysis is the application of generic sample preparation leading to sample extracts that are analysed by both LC-MS/MS and GC-MS/MS, this being nowadays one of the most "universal" approach in PRA.

\section{Acknowledgements}

This work forms part of the project P1 1B2010-23 (Universitat Jaume I- Fundació Bancaixa). The authors acknowledge the financial support from the Generalitat Valenciana, as a research group of excellence PROMETEO/2009/054. M.I. Cervera is very grateful to the Ministry of Economy and Competitivity for her predoctoral grant. 


\section{References}

1. V. Andreu and Y. Picó, Determination of currently used pesticides in biota, Anal. Bioanal. Chem., 2012, 404(9), 2659-2681.

2. H. V. Botitsi, S. D. Garbis, A. Economou and D. F. Tsipi, Current mass spectrometry strategies for the analysis of pesticides and their metabolites in food and water matrices, Mass Spectrom. Rev., 2011, 30(5), 907-939.

3. L. Mondello, P. Q. Tranchida, P. Dugo and G. Dugo, Comprehensive twodimensional gas chromatography-mass spectrometry: A review, Mass Spectrom. Rev., 2008, 27(2), 101-124.

4. T. Cajka and J. Hajslová, Gas chromatography-time-of-flight mass spectrometry in food analysis, LC.GC Eur., 2007, 20, 1.

5. M. I. Cervera, T. Portolés, E. Pitarch, J. Beltrán and F. Hernández, Application of gas chromatography time-of-flight mass spectrometry for target and non-target analysis of pesticide residues in fruits and vegetables, J. Chromatogr., A, 2012, 1244, 168-177.

6. F. Hernández, T. Portolés, E. Pitarch and F. J. López, Gas chromatography coupled to high-resolution time-of-flight mass spectrometry to analyze trace-level organic compounds in the environment, food safety and toxicology, TrAC, Trends Anal. Chem., 2011, 30(2), 388-400.

7. J. Haib, I. Hofer and J.-M. Renaud, Analysis of multiple pesticide residues in tobacco using pressurized liquid extraction, automated solid-phase extraction cleanup and gas chromatography-tandem mass spectrometry, J. Chromatogr., A, 2003, 1020(2), 173-187 .

8. K. Patel, R. J. Fussell, M. Hetmanski, D. M. Goodall and B. J. Keely, Evaluation of gas chromatography-tandem quadrupole mass spectrometry for the determination of organochlorine pesticides in fats and oils, J. Chromatogr., A, 2005, 1068(2), 289-296 .

9. C. C. Leandro, R. J. Fussell and B. J. Keely, Determination of priority pesticides in baby foods by gas chromatography tandem quadrupole mass spectrometry, $J$. Chromatogr., A, 2005,1085(2), 207-212.

10. A. G. Frenich, M. J. González-Rodríguez, F. J. Arrebola and J. L. Martínez Vidal, Potentiality of gas chromatography-triple quadrupole mass spectrometry in 
vanguard and rearguard methods of pesticide residues in vegetables, Anal. Chem., $2005,77,4640-4648$.

11. F. Hernández, T. Portolés, E. Pitarch, F. J. López, J. Beltrán and C. Vázquez, Potential of gas chromatography coupled to triple quadrupole mass spectrometry for quantification and confirmation of organohalogen xenoestrogen compounds in human breast tissues, Anal. Chem., 2005, 77, 7662-7672.

12. M. Mezcua, C. Ferrer, J. F. García-Reyes, M. J. Martínez-Bueno, M. Sigrist and A. R. Fernández-Alba, Analyses of selected non-authorized insecticides in peppers by gas chromatography/mass spectrometry and gas chromatography/tandem mass spectrometry, Food Chem., 2009, 112(1), 221225 .

13. A. G. Frenich, P. Plaza-Bolaños and J. L. M. Vidal, Comparison of tandem-inspace and tandem-in-time mass spectrometry in gas chromatography determination of pesticides: Application to simple and complex food samples, $J$. Chromatogr., A, 2008, 1203(2), 229-238 .

14. F. J. Santos and M. T. Galceran, Modern developments in gas chromatographymass spectrometry-based environmental analysis, J. Chromatogr., A, 2003, 1000(1-2), 125-151.

15. A. Kaufmann, The current role of high-resolution mass spectrometry in food analysis, Anal. Bioanal. Chem., 2011, 403(5), 1233-1249.

16. N. Belmonte Valles, M. Retamal, M. Mezcua and A. R. Fernández-Alba, A sensitive and selective method for the determination of selected pesticides in fruit by gas chromatography/mass spectrometry with negative chemical ionization, $J$. Chromatogr., A, 2012, 1264, 110-116.

17. J. Dong, Y. Pan, J. Lv, J. Sun, X. Gong and K. Li, Multiresidue method for the determination of pesticides in fruits and vegetables using gas chromatographynegative chemical ionization-triple quadrupole tandem mass spectrometry, Chromatographia, 2011, 74(1-2), 109-119.

18. T. Portolés, L. Cherta, J. Beltran and F. Hernández, Improved gas chromatography-tandem mass spectrometry determination of pesticide residues making use of atmospheric pressure chemical ionization, J. Chromatogr., A, 2012, 1260, 183-192 . 
19. T. Portolés, J. G. J. Mol, J. V. Sancho and F. Hernández, Advantages of atmospheric pressure chemical ionization in gas chromatography tandem mass spectrometry: Pyrethroid insecticides as a case study, Anal. Chem., 2012, 84(22), 9802-9810.

20. V. Nardelli, D. dell'Oro, C. Palermo and D. Centonze, Multi-residue method for the determination of organochlorine pesticides in fish feed based on a cleanup approach followed by gas chromatography-triple quadrupole tandem mass spectrometry, J. Chromatogr., A, 2010, 1217(30), 4996-5003 .

21. J. Sánchez-Avila, M. Fernandez-Sanjuan, J. Vicente and S. Lacorte, Development of a multi-residue method for the determination of organic micropollutants in water, sediment and mussels using gas chromatography-tandem mass spectrometry, J. Chromatogr., A, 2011, 1218(38), 6799-6811 .

22. E. Fuentes, M. E. Báez and A. Quiñones, Suitability of microwave-assisted extraction coupled with solid-phase extraction for organophosphorus pesticide determination in olive oil, J. Chromatogr., A, 2008, 1207(1-2), 38-45 .

23. E. Fuentes, M. E. Báez and J. Díaz, Microwave-assisted extraction at atmospheric pressure coupled to different cleanup methods for the determination of organophosphorus pesticides in olive and avocado oil, J. Chromatogr., A, 2009, 1216(51), 8859-8866 .

24. L.-J. Qu, H. Zhang, J.-H. Zhu, G.-S. Yang and H. Y. Aboul-Enein, Rapid determination of organophosphorous pesticides in leeks by gas chromatographytriple quadrupole mass spectrometry,Food Chem., 2010, 122(1), 327-332 .

25. M. L. Feo, E. Eljarrat and D. Barceló, Performance of gas chromatography/tandem mass spectrometry in the analysis of pyrethroid insecticides in environmental and food samples, Rapid Commun. Mass Spectrom., 2011, 25(7), 869-876.

26. J. Le Faouder, E. Bichon, P. Brunschwig, R. Landelle, F. Andre and B. Le Bizec, Transfer assessment of fipronil residues from feed to cow milk, Talanta, 2007, 73(4), 710-717.

27. A. Peruga, M. Barreda, J. Beltrán and F. Hernández, A robust GC-MS/MS method for the determination of chlorothalonil in fruits and vegetables, Food Addit. Contam., Part A, 2013, 30(2), 298-307. 
28. A. Garrido Frenich, J. L. Martínez Vidal, A. D. Cruz Sicilia, M. J. González Rodríguez and P. Plaza Bolaños, Multiresidue analysis of organochlorine and organophosphorus pesticides in muscle of chicken, pork and lamb by gas chromatography-triple quadrupole mass spectrometry, Anal. Chim. Acta, 2006, 558(1-2), 42-52.

29. A. Garrido-Frenich, R. Romero-González, J. L. Martínez-Vidal, P. Plaza-Bolaños, L. Cuadros-Rodríguez and M. A. Herrera-Abdo, Characterization of recovery profiles using gas chromatography-triple quadrupole mass spectrometry for the determination of pesticide residues in meat samples, J. Chromatogr., A, 2006, 1133(1-2), 315-321.

30. A. G. Frenich, P. P. Bolaños and J. L. M. Vidal, Multiresidue analysis of pesticides in animal liver by gas chromatography using triple quadrupole tandem mass spectrometry, J. Chromatogr., A, 2007, 1153(1-2), 194-202.

31. P. P. Bolaños, A. G. Frenich and J. L. M. Vidal, Application of gas chromatography-triple quadrupole mass spectrometry in the quantificationconfirmation of pesticides and polychlorinated biphenyls in eggs at trace levels, $J$. Chromatogr., A, 2007, 1167(1), 9-17.

32. Y.-G. Zhao, H. Shen, J. Shi, X. Chen and M. Jin, Preparation and characterization of amino functionalized nano-composite material and its application for multiresidue analysis of pesticides in cabbage by gas chromatography-triple quadrupole mass spectrometry, J. Chromatogr., A, 2011, 1218(33), 5568-5580.

33. J. L. Martínez Vidal, F. J. Arrebola Liébanas, M. J. González Rodríguez, A. Garrido Frenich and J. L. Fernández Moreno, Validation of a gas chromatography/triple quadrupole mass spectrometry based method for the quantification of pesticides in food commodities, Rapid Commun. Mass Spectrom., 2006, 20(3), 365-375.

34. S. Walorczyk and B. Gnusowski, Fast and sensitive determination of pesticide residues in vegetables using low-pressure gas chromatography with a triple quadrupole mass spectrometer, J. Chromatogr., A, 2006, 1128(1-2), 236-243.

35. M. Okihashi, S. Takatori, Y. Kitagawa and Y. Tanaka, Simultaneous analysis of 260 pesticide residues in agricultural products by gas chromatography/triple quadrupole mass spectrometry, J. AOAC Int., 2007, 90(4), 1165-1179. 
36. P. P. Bolaños, J. L. F. Moreno, D. D. Shtereva, A. G. Frenich and J. L. M. Vidal, Development and validation of a multiresidue method for the analysis of 151 pesticide residues in strawberry by gas chromatography coupled to a triple quadrupole mass analyzer, Rapid Commun. Mass Spectrom., 2007, 21, 22822294.

37. T. Pihlström, G. Blomkvist, P. Friman, U. Pagard and B.-G. Österdahl, Analysis of pesticide residues in fruit and vegetables with ethyl acetate extraction using gas and liquid chromatography with tandem mass spectrometric detection, Anal. Bioanal. Chem., 2007, 389(6), 1773-1789.

38. S. Walorczyk, Development of a multi-residue screening method for the determination of pesticides in cereals and dry animal feed using gas chromatography-triple quadrupole tandem mass spectrometry, J. Chromatogr., A, 2007, 1165(1-2), 200-212.

39. P. Payá, M. Anastassiades, D. Mack, I. Sigalova, B. Tasdelen, J. Oliva and A. Barba, Analysis of pesticide residues using the Quick Easy Cheap Effective Rugged and Safe (QuEChERS) pesticide multiresidue method in combination with gas and liquid chromatography and tandem mass spectrometric detection, Anal. Bioanal. Chem., 2007, 389(6), 1697-1714.

40. A. G. Frenich, J. L. F. Moreno, J. L. M. Vidal and F. J. A. Liébanas, Application of gas chromatography coupled to triple quadrupole mass spectrometry for the multiresidue analysis of pesticides in olive oil, J. Agric. Food Chem., 2007, 55(21), 8346-8352.

41. J. L. F. Moreno, A. G. Frenich, P. P. Bolaños and J. L. M. Vidal, Multiresidue method for the analysis of more than 140 pesticide residues in fruits and vegetables by gas chromatography coupled to triple quadrupole mass spectrometry, J. Mass Spectrom., 2008, 43(9), 1235-1254.

42. J. M. Lee, J. Park, G. Jang and K. Hwang, Comparative study of pesticide multiresidue extraction in tobacco for gas chromatography-triple quadrupole mass spectrometry, J. Chromatogr., A, 2008, 1187(1-2), 25-33.

43. S. Walorczyk, Development of a multi-residue method for the determination of pesticides in cereals and dry animal feed using gas chromatography-tandem quadrupole mass spectrometry. II. Improvement and extension to new analytes, $J$. Chromatogr., A, 2008, 1208(1-2), 202-214. 
44. S. Walorczyk, Application of gas chromatography/tandem quadrupole mass spectrometry to the multi-residue analysis of pesticides in green leafy vegetables, Rapid Commun. Mass Spectrom., 2008, 22(23), 3791-3801.

45. A. Garrido Frenich, J. L. Martínez Vidal, J. L. Fernández Moreno and R. RomeroGonzález, Compensation for matrix effects in gas chromatography-tandem mass spectrometry using a single point standard addition, J. Chromatogr., A, 2009, 1216(23), 4798-4808.

46. J. W. Wong, K. Zhang, K. Tech, D. G. Hayward, C. M. Makovi, A. J. Krynitsky, F. J. Schenck, K. Banerjee, S. Dasgupta and D. Brown, Multiresidue pesticide analysis in fresh produce by capillary gas chromatography-mass spectrometry/selective ion monitoring (GC-MS/SIM) and -tandem mass spectrometry (GC-MS/MS), J. Agric. Food Chem., 2010, 58(10), 5868-5883.

47. J. W. Wong, K. Zhang, K. Tech, D. G. Hayward, A. J. Krynitsky, I. Cassias, F. J. Schenck, K. Banerjee, S. Dasgupta and D. Brown, Multiresidue pesticide analysis of ginseng powders using acetonitrile- or acetone-based extraction, solid-phase extraction cleanup, and gas chromatography-mass spectrometry/selective ion monitoring (GC-MS/SIM) or -tandem mass spectrometry (GC-MS/MS), J. Agric. Food Chem., 2010, 58(10), 5884-5896.

48. M. I. Cervera, C. Medina, T. Portolés, E. Pitarch, J. Beltrán, E. Serrahima, L. Pineda, G. Muñoz, F. Centrich and F. Hernández, Multi-residue determination of 130 multiclass pesticides in fruits and vegetables by gas chromatography coupled to triple quadrupole tandem mass spectrometry, Anal. Bioanal. Chem., 2010, 397(7), 2873-2891.

49. F. J. Camino-Sánchez, A. Zafra-Gómez, J. Ruiz-García, R. Bermúdez-Peinado, O. Ballesteros, A. Navalon and J. L. Vílchez, UNE-EN ISO/IEC 17025:2005 accredited method for the determination of 121 pesticide residues in fruits and vegetables by gas chromatography-tandem mass spectrometry, J. Food Compos. Anal., 2011, 84(7), 869-881.

50. S. Walorczyk and D. Drozdzyński, Development and validation of a routine multiresidue method for determining 140 pesticides in fruits and vegetables by gas chromatography/tandem quadrupole mass spectrometry, J. AOAC Int., 2011, 94(5), 1625-1642. 
51. S. Walorczyk, D. Drodyński and B. Gnusowski, Multiresidue determination of 160 pesticides in wines employing mixed-mode dispersive-solid phase extraction and gas chromatography-tandem mass spectrometry, Talanta, 2011, 85(4), 18561870.

52. U. Koesukwiwat, S. J. Lehotay and N. Leepipatpiboon, Fast, low-pressure gas chromatography triple quadrupole tandem mass spectrometry for analysis of 150 pesticide residues in fruits and vegetables, J. Chromatogr., A, 2011, 1218(39), 7039-7050.

53. J. Robles-Molina, B. Gilbert-López, J. F. García-Reyes, N. R. Martos and A. Molina-Díaz, Multiclass determination of pesticides and priority organic pollutants in fruit-based soft drinks by headspace solid-phase microextraction/gas chromatography tandem mass spectrometry, Anal. Methods, 2011, 3(10), 22212230.

54. J. Zhao, B. Yao, J. Huang, X. Kong and A. Yue, A multi-residue method for fast determination of 78 pesticides in cranberry extract by gas chromatography triple quadripole mass spectrometry with quechers sample preparation, ISWREP - Proc. -Int. Symp. Water Resour. Environ. Prot., 2011, vol. 3, pp. 2084-2087.

55. S. Walorczyk, Gas chromatographic-tandem mass spectrometric analysis of pesticides residues in produce using concurrent solvent recondensation-large volume injection, J. Chromatogr., A, 2012, 1222, 98-108.

56. K.-G. Lee and E.-K. Jo, Multiresidue pesticide analysis in Korean ginseng by gas chromatography-triple quadrupole tandem mass spectrometry, Food Chem., 2012, 134(4), 2497-2503.

57. K. Banerjee, S. Utture, S. Dasgupta, C. Kandaswamy, S. Pradhan, S. Kulkarni and P. Adsule, Multiresidue determination of 375 organic contaminants including pesticides, polychlorinated biphenyls and polyaromatic hydrocarbons in fruits and vegetables by gas chromatography-triple quadrupole mass spectrometry with introduction of semi-quantification approach, J. Chromatogr., A, 2012, 1270, $283-295$.

58. Y. Sapozhnikova and S. J. Lehotay, Multi-class, multi-residue analysis of pesticides, polychlorinated biphenyls, polycyclic aromatic hydrocarbons, polybrominated diphenyl ethers and novel flame retardants in fish using fast, low- 
pressure gas chromatography-tandem mass spectrometry, Anal. Chim. Acta, $2013, \mathbf{7 5 8}, 80$.

59. Q. Lin, H. Shi and P. Xue, MSPD-GC-MS-MS determination of residues of 15 organic nitrogen-containing pesticides in vegetables, Chromatographia, 2010, 72(11-12), 1143-1148.

60. M. Anastassiades, S. J. Lehotay, D. Štajnbaher and F. J. Schenck, Fast and easy multiresidue method employing acetonitrile extraction/partitioning and "dispersive solid-phase extraction" for the determination of pesticide residues in produce, J. AOAC Int., 2003, 86(2), 412-431.

61. S. J. Lehotay, K. Maštovská and A. R. Lightfield, Use of buffering and other means to improve results of problematic pesticides in a fast and easy method for residue analysis of fruits and vegetables, J. AOAC Int., 2005, 88(2), 615-629.

62. K. Grob and M. Biedermann, The two options for sample evaporation in hot GC injectors: thermospray and band formation. Optimization of conditions and injector design, Anal. Chem., 2002, 74 (1), 10-16.

63. Commission Decision 2002/657/EC, 12 August 2002 implementing Council Directive 96/23/EC concerning the performance of analytical methods and the interpretation of results, L221 (2002/657/EC), Off. J. Eur. Comm., 2002, 221, p. 8.

64. SANCO/12495/2011. Method Validation and Quality Control Procedures for Pesticide Residues Analysis in Food and Feed, European Commission, Directorate General Health and Consumer Protection, 1 January, 2012.

65. E. Pitarch, C. Medina, T. Portolés, F. J. López and F. Hernández, Determination of priority organic micro-pollutants in water by gas chromatography coupled to triple quadrupole mass spectrometry, Anal. Chim. Acta, 2007, 583(2), 246-258.

66. A. Penetra, V. Vale Cardoso, E. Ferreira and M. J. Benoliel, Solid-phase extraction and gas chromatography-tandem mass spectrometry method for the simultaneous determination of several pesticides in water, Water Sci. Technol., 2010, 62(3), 667-675.

67. N. Barco-Bonilla, R. Romero-González, P. Plaza-Bolaños, A. Garrido Frenich and J. L. Martínez Vidal, Analysis and study of the distribution of polar and nonpolar pesticides in wastewater effluents from modern and conventional treatments, J. Chromatogr., A, 2010, 1217(50), 7817-7825. 
68. E. Pitarch, T. Portolés, J. M. Marín, M. Ibáñez, F. Albarrán and F. Hernández, Analytical strategy based on the use of liquid chromatography and gas chromatography with triple-quadrupole and time-of-flight MS analyzers for investigating organic contaminants in wastewater, Anal. Bioanal. Chem., 2010, 397(7), 2763-2776.

69. J. L. M. Vidal, J. A. P. Sánchez, P. Plaza-Bolaños, A. G. Frenich and R. RomeroGonzález, Use of pressurized liquid extraction for the simultaneous analysis of 28 polar and 94 non-polar pesticides in agricultural soils by GC/QqQ-MS/MS and UPLC/QqQ-MS/MS, J. AOAC Int., 2010, 93(6), 1715-1731.

70. F. J. Camino-Sánchez, A. Zafra-Gómez, J. P. Pérez-Trujillo, J. E. CondeGonzález, J. C. Marques and J. L. Vílchez, Validation of a GC-MS/MS method for simultaneous determination of 86 persistent organic pollutants in marine sediments by pressurized liquid extraction followed by stir bar sorptive extraction, Chemosphere, 2011, 84(7), 869-881.

71. C. Coscollà, M. Castillo, A. Pastor and V. Yusà, Determination of 40 currently used pesticides in airborne particulate matter (PM 10) by microwave-assisted extraction and gas chromatography coupled to triple quadrupole mass spectrometry, Anal. Chim. Acta, 2011, 693(1-2), 72-81.

72. A. Garrido Frenich, R. Romero-González, J. L. Martínez Vidal, R. Martínez Ocaña and P. Baquero Feria, Comparison of solid phase microextraction and hollow fiber liquid phase microextraction for the determination of pesticides in aqueous samples by gas chromatography triple quadrupole tandem mass spectrometry, Anal. Bioanal. Chem., 2010, 399(6), 2043-2059.

73. F. J. Camino-Sánchez, A. Zafra-Gómez, S. Cantarero-Malagón and J. L. Vílchez, Validation of a method for the analysis of 77 priority persistent organic pollutants in river water by stir bar sorptive extraction in compliance with the European Water Framework Directive, Talanta, 2012, 89, 322-334 .

74. F. J. Camino-Sánchez, A. Zafra-Gómez, J. Ruiz-García and J. L. Vílchez, Screening and quantification of 65 organic pollutants in drinking water by stir bar sorptive extraction-gas chromatography-triple quadrupole mass spectrometry, Food Anal. Methods, 2012, 6, 854-867.

75. J. Robles-Molina, B. Gilbert-López, J. F. García-Reyes and A. Molina-Díaz, Gas chromatography triple quadrupole mass spectrometry method for monitoring 
multiclass organic pollutants in Spanish sewage treatment plants effluents, Talanta, 2013, 111, 196-205 .

76. A. Rashid, S. Nawaz, H. Barker, I. Ahmad and M. Ashraf, Development of a simple extraction and cleanup procedure for determination of organochlorine pesticides in soil using gas chromatography-tandem mass spectrometry, $J$. Chromatogr., A, 2010, 1217(17), 2933-2939.

77. N. S. Chary, S. Herrera, M. J. Gómez and A. R. Fernández-Alba, Parts per trillion level determination of endocrine-disrupting chlorinated compounds in river water and wastewater effluent by stir-bar-sorptive extraction followed by gas chromatography-triple quadrupole mass spectrometry, Anal. Bioanal. Chem., 2012, 404, 1993-2006.

78. C. Devos, F. David and P. Sandra, A new validated analytical method for the determination of tributyltin in water samples at the quantification level set by the European Union, J. Chromatogr., A, 2012, 1261, 151-157.

79. B. Cavalliere, M. Monteleone, A. Naccarato, G. Sindona and A. Tagarelli, A solid-phase microextraction-gas chromatography approach combined with triple quadrupole mass spectrometry for the assay of carbamate pesticides in water samples, J. Chromatogr., A, 2012, 1257, 149-157.

80. M. I. Cervera, J. Beltran, F. J. Lopez and F. Hernandez, Determination of volatile organic compounds in water by head-space-solid-phase microextraction gas chromatography coupled to tandem mass spectrometry with triple quadrupole analyzer, Anal. Chim. Acta, 2011, 704, 87-97.

81. U. Koesukwiwat, S. J. Lehotay and N. Leepipatpiboon, Fast, low-pressure gas chromatography triple quadrupole tandem mass spectrometry for analysis of 150 pesticide residues in fruits and vegetables, J. Chromatogr., A, 2011, 1218(39), 7039-7050.

82. L. Cherta, J. Beltran, T. Portolés and F. Hernández, Multiclass determination of 66 organic micropollutants in environmental water samples by fast gas chromatography-mass spectrometry, Anal. Bioanal. Chem., 2011, 402(7), 23012314.

83. Y. Sapozhnikova and S. J. Lehotay, Multi-class, multi-residue analysis of pesticides, polychlorinated biphenyls, polycyclic aromatic hydrocarbons, polybrominated diphenyl ethers and novel flame retardants in fish using fast, low- 
pressure gas chromatography-tandem mass spectrometry, Anal. Chim. Acta, 2013, 758, 80-92.

84. R. Húšková, E. Matisová, S. Hrouzková and L. Švorc, Analysis of pesticide residues by fast gas chromatography in combination with negative chemical ionization mass spectrometry, J. Chromatogr., A, 2009, 1216(35), 6326-6334 . 